\title{
Electrostatic potential variations on stellarator magnetic surfaces in low collisionality regimes
}

\author{
Iván Calvo $^{1, \dagger}$, José Luis Velasco ${ }^{1}$, Félix I. Parra ${ }^{2,3}$, J. Arturo Alonso ${ }^{1}$ and \\ José Manuel García-Regaña ${ }^{1}$ \\ ${ }^{1}$ Laboratorio Nacional de Fusión, CIEMAT, 28040 Madrid, Spain \\ ${ }^{2}$ Rudolf Peierls Centre for Theoretical Physics, University of Oxford, Oxford OX1 3PU, UK \\ ${ }^{3}$ Culham Centre for Fusion Energy, Abingdon OX14 3DB, UK
}

(Received 28 May 2018; revised 12 July 2018; accepted 12 July 2018)

The component of the neoclassical electrostatic potential that is non-constant on the magnetic surface, that we denote by $\tilde{\varphi}$, can affect radial transport of highly charged impurities, and this has motivated its inclusion in some modern neoclassical codes. The number of neoclassical simulations in which $\tilde{\varphi}$ is calculated is still scarce, partly because they are usually demanding in terms of computational resources, especially at low collisionality. In this paper the size, the scaling with collisionality and with aspect ratio and the structure of $\tilde{\varphi}$ on the magnetic surface are analytically derived in the $1 / v, \sqrt{v}$ and superbanana-plateau regimes of stellarators close to omnigeneity; i.e. stellarators that have been optimized for neoclassical transport. It is found that the largest $\tilde{\varphi}$ that the neoclassical equations admit scales linearly with the inverse aspect ratio and with the size of the deviation from omnigeneity. Using a model for a perturbed omnigenous configuration, the analytical results are verified and illustrated with calculations by the code KNOSOS. The techniques, results and numerical tools employed in this paper can be applied to neoclassical transport problems in tokamaks with broken axisymmetry.

Key words: fusion plasma, plasma confinement

\section{Introduction}

The transport of impurities in three-dimensional toroidal magnetic fields has attracted much attention from the stellarator community. In stellarators, the accumulation of impurities in the centre of the confinement region often limits the discharge duration and is considered to be a potential problem for the development of future reactors. In the framework of neoclassical theory, this accumulation has generally been explained with the inward convection caused by the typically negative radial electric field acting on the highly charged ions in the absence of a so-called 'temperature screening' effect in non-axisymmetric systems. ${ }^{1}$ In a qualitative sense,

$\dagger$ Email address for correspondence: ivan.calvo@ciemat.es

${ }^{1}$ Recently, both the prevalence of the radial electric field in the transport of impurities and the absence of impurity screening in three-dimensional magnetic fields have been brought into question for several collisionality regimes (Velasco et al. 2017; Helander et al. 2017). 
these expectations are consistent with the general trend observed in the impurity confinement time (Burhenn et al. 2009), although there are remarkable experimental observations of outward impurity transport (see, for example, McCormick et al. 2002, Ida et al. 2009). This outward impurity flux is still poorly understood. On a quantitative level, it is difficult to determine whether the observed impurity fluxes agree with calculations relying on the solution of approximate versions of the drift-kinetic equation, for these comparisons are often fragmented, dealing with a reduced number of plasma profiles and based on different measuring techniques. The fundamental output of neoclassical modelling of impurity transport is the spatially resolved radial particle flux of a charge state of a certain impurity species, and it is often the case that this very quantity is not experimentally accessible, which complicates the direct quantitative comparisons.

The kinetic modelling of impurity dynamics and transport has undergone recent improvements with the inclusion of terms that had previously been considered of secondary importance (García-Regaña et al. 2013). These terms model the modification of the trajectories of impurity ions by the component of the electrostatic potential that is non-constant on the flux surface, that we denote by $\tilde{\varphi}$ and that defines the component of the electric field that is tangent to the flux surface. Specifically, this tangential electric field produces a radial $E \times B$ drift that advects impurities on the flux surface. The effect of $\tilde{\varphi}$ on impurity transport is larger for impurities with higher electric charge.

A previous step to the assessment of the impact of $\tilde{\varphi}$ on radial impurity transport is the neoclassical calculation of $\tilde{\varphi}$ itself $^{2}$, which has typically been ignored in standard neoclassical simulations. The modern codes EUTERPE (Kornilov et al. 2005) and SFINCS (Landreman et al. 2014) can compute it. In García-Regaña et al. (2017), results of $\tilde{\varphi}$ from both codes for different stellarators and plasma regimes are presented. These extended neoclassical models have started to be used to revisit some of the previous calculations of neoclassical impurity fluxes and have shown substantial deviations (with respect to codes that do not include the effect of $\tilde{\varphi}$ ) for some machines and plasma conditions (García-Regaña et al. 2017; Mollén et al. 2018). Recently, the strong effect of $\tilde{\varphi}$ on the neoclassical transport of highly charged collisional impurities in stellarators has been analytically proven in (Calvo et al. 2018).

Given the relevance of correctly determining $\tilde{\varphi}$, it is important to understand the equations that have to be solved to calculate it, and to derive and discuss analytically some features of the solutions to these equations. In this paper we do so for main ions in the $1 / v, \sqrt{v}$ and superbanana-plateau low collisionality regimes (see Mynick 1984 for estimations of the effect of $\tilde{\varphi}$ on main ion transport in low collisionality regimes under some simplified assumptions). Low collisionality regimes are the pertinent ones for the main ions in the core of reactor-relevant stellarator plasmas and, in addition, in these regimes $\tilde{\varphi}$ is large and has significant impact on impurity transport. We will carry out such analytical discussion by using the techniques and results of Calvo et al. (2017), where we studied the radial neoclassical transport of low collisionality ions in stellarators close to omnigeneity. Omnigenous magnetic fields (Hall \& McNamara 1975; Cary \& Shasharina 1997; Parra et al. 2015) are those for which the orbit-averaged radial magnetic drift vanishes for all trapped particles. Omnigenous stellarators can be said to be perfectly optimized from a neoclassical point of view, exhibiting transport levels similar to those of a tokamak. In Calvo

\footnotetext{
${ }^{2}$ As a matter of fact, this has brought up the relevance of the measurement of $\tilde{\varphi}$ (Pedrosa et al. 2015) and its impact on impurity density inhomogeneities (Arévalo et al. 2014; Alonso et al. 2016).
} 
et al. (2017), expansions in large aspect ratio were not considered. In this article, we expand in small inverse aspect ratio (in the end, all stellarators in operation today have large aspect ratio (Beidler et al. 2011)). We will see that the large aspect ratio expansion introduces a number of subtleties.

Let us emphasize that closeness to omnigeneity is not a mere academic requirement. It is well-known (Galeev \& Sagdeev 1979; Ho \& Kulsrud 1987) that, for sufficiently low ion collisionality, tangential drifts have to be kept in the stellarator drift-kinetic equation. If the radial electric field is not small and the aspect ratio is large, one can show that the drift kinetic equation is radially local and that the tangential magnetic drift is negligible compared to the tangential $E \times B$ drift. But if one of these assumptions fails (that is, if the radial electric field is small or the stellarator is compact), the drift-kinetic equation becomes radially global (with obvious negative consequences for confinement, because then the radial excursion associated with a particle orbit can be as large as the stellarator minor radius) at small collisionality values unless the stellarator is close to omnigeneity. If the magnetic configuration is close to being omnigenous and the deviation from omnigeneity has small spatial gradients, the set of equations consisting of the drift-kinetic equation and the quasineutrality equation can be rigorously proven to be linear and radially local (Calvo et al. 2017). Not only do the resulting equations include the effect of $\tilde{\varphi}$, but also the effect of the tangential magnetic drift. As we have pointed out, the tangential magnetic drift has to be taken into account to describe the transport of the main ions at very low collisionality in compact stellarators and in situations of small radial electric field even if the aspect ratio is large. The importance of the tangential magnetic drift in the latter case is a subject of active research (Matsuoka et al. 2015). In this paper, we will pay special attention to the behaviour of $\tilde{\varphi}$ in regimes where the tangential magnetic drift has to be retained.

Apart from discussing $\tilde{\varphi}$ analytically in the $1 / v, \sqrt{v}$ or superbanana-plateau regimes, we will verify and illustrate the results by comparing them with KNOSOS (Velasco et al. 2018), a newly developed bounce-averaged code that solves the drift-kinetic and quasineutrality equations derived in Calvo et al. (2017) (for the time being, KNOSOS uses a pitch-angle scattering collision operator), and therefore retains the tangential magnetic drift (among the two codes mentioned some paragraphs above, EUTERPE does not retain the tangential magnetic drift, whereas a way to try to retain it in SFINCS has been recently reported in Paul et al. 2017). Finally, a remark on the scope of this paper is in order. Whereas the expansion around omnigeneity should be considered as a realistic approach to the analysis of magnetic configurations that aspire to confine sufficiently well to be the basis of stellarator reactor designs, our present treatment is limited by the requirement of small gradients of the non-omnigenous perturbation in Calvo et al. (2017). It would be important to address the relaxation of this condition in future research. Some ideas about the kind of complications introduced by large gradients can be found in Calvo et al. (2014, 2015) in the setting of perturbed quasisymmetric configurations. Stellarators close to quasisymmetry, where the non-quasisymmetric perturbation has small gradients, were treated in detail in Calvo et al. (2013). Finally, we note that a tokamak with (slightly) broken axisymmetry can be viewed as a particular case of a magnetic configuration close to omnigeneity, and therefore the tools and results of this paper can be directly applied to problems such as the calculation of tokamak neoclassical toroidal viscosity.

The rest of the paper is organized as follows. In $\S 2$, after defining the coordinates that will be employed in the theoretical derivations $(\$ 2.1)$ and introducing the notion of omnigeneity $(\S 2.2)$, we present in $\S 2.3$ the neoclassical equations that determine 
$\tilde{\varphi}$ in low collisionality regimes of stellarators close to omnigeneity when the deviation from omnigeneity has small spatial gradients. We will also see how $\tilde{\varphi}$ scales with the size of the deviation from omnigeneity. In $\S 2.4$, and for large aspect ratio, the scaling with aspect ratio of some quantities entering the neoclassical equations is given; these expressions will be useful in later sections. In $\S 2.5$, the magnetic field close to omnigeneity that we will use for the numerical examples is described. With this magnetic field, and taking reactor-relevant parameter values, in $\S 3$ we give a plot (see figure 3) calculated by KNOSOS showing the dependence of the size of the non-constant component of the electrostatic potential on the collisionality and the radial electric field. The objective of the next section, $\S 4$, is to explain mathematically the different regimes observed in plots like that in figure 3 . Hence, in $\$ 4$ we discuss analytically the features of $\tilde{\varphi}$ in the $1 / v, \sqrt{v}$ and superbanana-plateau asymptotic regimes as implied by the equations given in $\S 2.3$ (as explained in Calvo et al. 2017, those equations are not valid for lower collisionality regimes like the superbanana or $v$ regimes, the essential reason being that for such low collisionality values one has to take into account the radial displacement of the trajectories). In particular, we will comment on the scaling of $\tilde{\varphi}$ with collisionality and aspect ratio, as well as on its behaviour under stellarator symmetry transformations. Detailed numerical checks of the analytical results with KNOSOS are provided throughout this section. Of course, assuming closeness to omnigeneity is not required if one is only interested in studying the $1 / v$ and $\sqrt{v}$ regimes (the former exists in any stellarator and the latter exists in large aspect ratio stellarators with non-small radial electric field), but the assumption is necessary to be able to study, in the same framework, those two collisionality regimes together with the superbanana-plateau regime. In $\$ 5$, we give the conclusions. In particular, table 1 condenses the main analytical results derived in the paper. Finally, we point out that, for simplicity, throughout the paper we assume that the electrons are adiabatic. This is a good approximation in many cases, but its accuracy certainly depends on the particular collisionality regimes in which the main ions and the electrons are, and situations in which the electrons have to be treated kinetically are not uncommon (see e.g. Velasco et al. 2017, García-Regaña et al. 2018). In these situations, a kinetic treatment of the electrons is also needed to calculate $\tilde{\varphi}$.

\section{Low collisionality neoclassical equations in stellarators close to omnigeneity}

\subsection{Phase space coordinates}

In the kinetic description of low collisionality regimes, it is useful to employ spatial coordinates $\{r, \alpha, l\}$, defined as follows. The radial coordinate $r \in[0, a]$ labels the magnetic surface and has dimensions of length, $a$ being the minor radius of the stellarator. For given $r$, the angular coordinate $\alpha \in[0,2 \pi)$ selects a magnetic field line on the surface. The coordinate $l \in\left[0, l_{\max }(r, \alpha)\right)$ is the arc length along the field line. In these coordinates the magnetic field reads

$$
\boldsymbol{B}=\Psi(r) \nabla r \times \nabla \alpha .
$$

As for the meaning of the flux function $\Psi(r)$, it is easy to check that $2 \pi \int_{0}^{r_{0}} \Psi(r) \mathrm{d} r$ equals the magnetic flux across a surface defined by $l=$ const. and $0 \leqslant r \leqslant r_{0}$.

In these coordinates the flux-surface average of a function $f(r, \alpha, l)$ is defined as

$$
\langle f\rangle(r)=V^{\prime}(r)^{-1} \int_{0}^{2 \pi} \mathrm{d} \alpha \int_{0}^{l_{\max }} \mathrm{d} l \Psi B^{-1} f,
$$




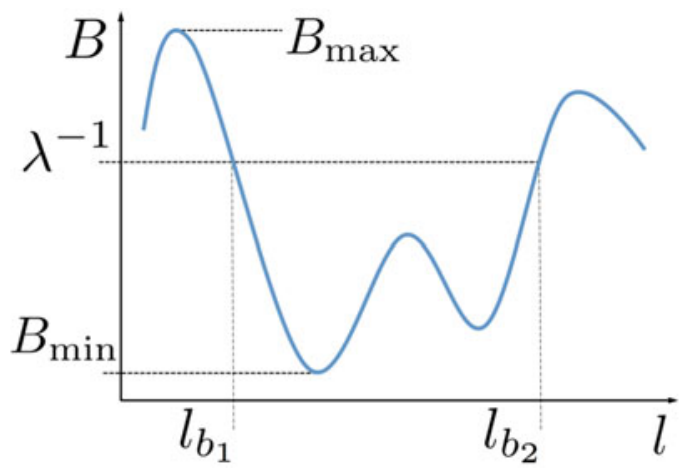

FIGURE 1. Cartoon showing some of the quantities (defined in the text) employed to describe a trapped trajectory.

where $\Psi B^{-1}=[(\nabla r \times \nabla \alpha) \cdot \nabla l]^{-1}$ is the volume element (the coordinates $\{r, \alpha, l\}$ are chosen such that $\Psi>0), B(r, \alpha, l)$ is the magnitude of $\boldsymbol{B}$ and

$$
V^{\prime}(r)=\int_{0}^{2 \pi} \mathrm{d} \alpha \int_{0}^{l_{\max }} \mathrm{d} l \Psi B^{-1},
$$

with $V(r)$ being the volume enclosed by the surface $r$. We will use primes to denote differentiation with respect to $r$.

In velocity space, we employ coordinates $\{v, \lambda, \sigma\}$, where $v$ is the magnitude of the velocity $\boldsymbol{v}, \lambda=v_{\perp}^{2} /\left(B v^{2}\right)$ is the pitch-angle coordinate, $v_{\perp}$ is the component of $\boldsymbol{v}$ perpendicular to $\boldsymbol{B}$ and $\sigma=v_{\|} /\left|v_{\|}\right|$is the sign of the parallel velocity, with

$$
v_{\|}(r, \alpha, l, v, \lambda, \sigma)=\sigma v \sqrt{1-\lambda B(r, \alpha, l)} .
$$

Integrals over velocity space of gyrophase-independent functions (all functions in this paper are independent of gyrophase) read, in these coordinates,

$$
\int(\cdot) \mathrm{d}^{3} v \equiv \sum_{\sigma} \int_{0}^{\infty} \mathrm{d} v \int_{0}^{B^{-1}} \mathrm{~d} \lambda \frac{\pi B v^{3}}{\left|v_{\|}\right|}(\cdot) .
$$

\subsection{Perturbed omnigenous magnetic fields}

Trapped trajectories are those with $\lambda \in\left[B_{\max }^{-1}, B_{\min }^{-1}\right]$, where $B_{\max }(r)$ and $B_{\min }(r)$ are, respectively, the maximum and minimum values of $B$ on the flux surface. For each trapped trajectory, the second adiabatic invariant reads

$$
J(r, \alpha, v, \lambda)=2 \int_{l_{b_{1}}}^{l_{b_{2}}}\left|v_{\|}\right| \mathrm{d} l .
$$

Here, $l_{b_{1}}$ and $l_{b_{2}}$ are the bounce points of the trapped orbit, obtained by solving in $l$ the equation $1-\lambda B(r, \alpha, l)=0$ (see figure 1 for a cartoon showing some of the quantities that describe trapped trajectories).

The importance of $J$ comes from the relations

$$
\frac{2}{\tau_{b}} \int_{l_{b_{1}}}^{l_{b_{2}}} \frac{1}{\left|v_{\|}\right|} \boldsymbol{v}_{M} \cdot \nabla r \mathrm{~d} l=\frac{m}{Z e \Psi \tau_{b}} \partial_{\alpha} J,
$$




$$
\frac{2}{\tau_{b}} \int_{l_{b_{1}}}^{l_{b_{2}}} \frac{1}{\left|v_{\|}\right|} \boldsymbol{v}_{M} \cdot \nabla \alpha \mathrm{d} l=-\frac{m}{Z e \Psi \tau_{b}} \partial_{r} J
$$

where

$$
\boldsymbol{v}_{M}=\frac{m v^{2}(1-\lambda B)}{Z e B} \hat{\boldsymbol{b}} \times(\hat{\boldsymbol{b}} \cdot \nabla \hat{\boldsymbol{b}})+\frac{m v^{2} \lambda}{2 Z e B} \hat{\boldsymbol{b}} \times \nabla B
$$

is the magnetic drift, $\hat{\boldsymbol{b}}=B^{-1} \boldsymbol{B}, m$ is the particle mass, $Z e$ is the particle charge (we assume $Z \sim 1$ ), $e$ is the proton charge and

$$
\tau_{b}(r, \alpha, v, \lambda)=2 \int_{l_{b_{1}}}^{l_{b_{2}}}\left|v_{\|}\right|^{-1} \mathrm{~d} l
$$

is the bounce time; i.e. the time that the trapped particle needs to complete its orbit. Hence, $\partial_{\alpha} J$ and $\partial_{r} J$ contain the information on the motion of the trapped particle in the directions perpendicular to $\boldsymbol{B}$, averaged over the motion in the direction parallel to $B$.

The magnetic field is called omnigenous (Cary \& Shasharina 1997; Parra et al. $2015)$ if the average radial magnetic drift vanishes for all trapped particles. Due to (2.7), this is equivalent to saying that the second adiabatic invariant is independent of $\alpha, \partial_{\alpha} J=0$, for all trapped trajectories.

From the definition of omnigeneity one can deduce that, for any function $q$ that depends on $\alpha$ and $l$ only through an omnigenous magnetic field $B$, one has (Cary $\&$ Shasharina 1997; Helander \& Nührenberg 2009; Calvo et al. 2017)

$$
\partial_{\alpha} \int_{l_{b_{1}}}^{l_{b_{2}}} q(r, v, \lambda, B(r, \alpha, l)) \mathrm{d} l=0 .
$$

This useful property is employed in the derivation of the neoclassical equations of $\S 2.3$ and it will also be used in the analysis of their solutions.

In this paper, we will carry out the calculations in magnetic configurations that can be written as an exactly omnigenous magnetic field $\boldsymbol{B}_{0}$ plus a perturbation $\delta \boldsymbol{B}_{1}$,

$$
\boldsymbol{B}=\boldsymbol{B}_{0}+\delta \boldsymbol{B}_{1},
$$

where $B_{0}:=\left|\boldsymbol{B}_{0}\right| \sim\left|\boldsymbol{B}_{1}\right|=: B_{1}$ and $0 \leqslant \delta \ll 1$. As advanced in $\S 1$, and following Calvo et al. (2017), we restrict ourselves to cases in which the characteristic scale of variation of $B_{1}$ on the flux surface is not much smaller than that of $B_{0}$. Specifically, we require $\left|\partial_{\alpha} B_{1}\right| /\left|\partial_{\alpha} B_{0}\right| \sim\left|\partial_{l} B_{1}\right| /\left|\partial_{l} B_{0}\right| \ll \delta^{-1}$. Thus, in terms of an expansion in $\delta \ll 1$, we can assume that $B_{0}$ and $B_{1}$ have a similar characteristic variation length on the flux surface, that we can take to be $R_{0}$, the major radius of the stellarator. This is what we mean when we say that the deviation from omnigeneity has small gradients. In particular, deviations with small gradients can deform the omnigenous magnetic wells, but not create new wells.

\subsection{Drift-kinetic and quasineutrality equations in stellarators close to omnigeneity}

In drift kinetics (Hazeltine 1973; Parra \& Calvo 2011; Calvo et al. 2013), the characteristic frequency of particle motion in the direction parallel to $\boldsymbol{B}$ is $O\left(v_{t} / R_{0}\right)$, whereas the motion perpendicular to the magnetic field has a much smaller typical frequency $O\left(\rho_{*} v_{t} / R_{0}\right)$, where $\rho_{*}=\rho / R_{0} \ll 1$ is the normalized Larmor radius, with 
$\rho=v_{t} / \Omega$ and $\Omega=Z e B / m$. Low collisionality regimes ${ }^{3}$ are defined by $v_{*} \ll 1$, where $v_{*}=v_{i i} R_{0} / v_{t}$ is the ion collisionality, $v_{i i}$ is the ion-ion collision frequency and $v_{t}=\sqrt{T / m}$ is the ion thermal speed. The condition $v_{*} \ll 1$ means that the characteristic parallel streaming frequency is much greater than the collision frequency $\nu_{i i}$, and therefore the drift-kinetic ion equation can be averaged over the parallel motion. In this subsection, we give the neoclassical equations for low collisionality plasmas in stellarators close to omnigeneity where the deviation from omnigeneity has small gradients, but we do not include their derivation. For that, we refer the reader to Calvo et al. (2017). No assumptions are made about the aspect ratio of the stellarator yet.

For $\delta \ll 1$, one finds that the electrostatic potential, $\varphi$, is a flux function to lowest order,

$$
\varphi(r, \alpha, l)=\varphi_{0}(r)+\delta \varphi^{(1)}(r, \alpha, l)+\cdots .
$$

The component of the electrostatic potential that is non-constant on the flux surface, denoted in $\S 1$ by $\tilde{\varphi}$, is therefore given by

$$
\tilde{\varphi}=\varphi-\varphi_{0}=\delta \varphi^{(1)}+\cdots .
$$

In an expansion in $\delta$, the ion distribution function, $F$, can be written as

$$
F=F_{M}-\frac{Z e \delta \varphi^{(1)}}{T} F_{M}+\delta g^{(1)}+\cdots,
$$

where $F_{M}$ is a Maxwellian distribution constant on flux surfaces,

$$
F_{M}(r, v)=n(r)\left(\frac{m}{2 \pi T(r)}\right)^{3 / 2} \exp \left(-\frac{m v^{2}}{2 T(r)}\right),
$$

and $n$ and $T$ are the ion density and temperature. The non-adiabatic perturbation to the Maxwellian distribution, $g^{(1)}(r, \alpha, l, v, \lambda)$, is independent of $l$ and $\sigma$, and vanishes for passing trajectories of the omnigenous field, $0 \leqslant \lambda<B_{0, \max }^{-1}$, where $B_{0, \max }(r)$ is the maximum value of $B_{0}$ on the flux surface. The function $g^{(1)}$ can be chosen such that

$$
\int_{0}^{2 \pi} g^{(1)} \mathrm{d} \alpha=0
$$

The equations that determine $g^{(1)}$ and $\varphi^{(1)}$ are radially local and linear. First, one has the bounce-averaged drift-kinetic equation for trapped trajectories,

$$
-\partial_{r} J^{(0)} \partial_{\alpha} g^{(1)}+\partial_{\alpha} J^{(1)} \Upsilon F_{M}=\sum_{\sigma} \frac{Z e \Psi}{m} \int_{l_{b_{10}}}^{l_{b_{20}}} \frac{1}{\left|v_{\|}^{(0)}\right|} C_{i i}^{\ell(0)}\left[g^{(1)}\right] \mathrm{d} l,
$$

where $^{4}$

$$
\partial_{r} J^{(0)}=-\int_{l_{b_{10}}}^{l_{b_{20}}} \frac{\lambda v \partial_{r} B_{0}+2 Z e /(m v) \varphi_{0}^{\prime}}{\sqrt{1-\lambda B_{0}}} \mathrm{~d} l,
$$

\footnotetext{
${ }^{3}$ From here on, we understand that quantities without a subindex specifying the species refer to ions. Electron quantities will always include a subindex $e$. In some ion quantities where some ambiguity might exist, as in $v_{i i}$, we include subindices.

${ }^{4}$ The presence of $\varphi$ in the expressions for $\partial_{r} J^{(0)}$ and $J^{(1)}$ might seem surprising in the light of the discussion on the second adiabatic invariant in $\S 2.2$. When $\varphi$ is non-zero, the average tangential and radial drifts (that now include the corresponding components of the $E \times B$ drift) are obtained by taking derivatives with respect to $r$ and $\alpha$ keeping $\mathcal{E}=m v^{2} / 2+Z e \varphi / m$ and $\mu=v_{\perp}^{2} /(2 B)$ constant. After this, one can perform the $\delta$ expansion and change to coordinates $v$ and $\lambda$, obtaining (2.19) and (2.20). This process is explained step by step in Calvo et al. (2017).
} 


$$
\begin{gathered}
J^{(1)}=-\int_{l_{b_{10}}}^{l_{b_{20}}} \frac{\lambda v B_{1}+2 Z e /(m v) \varphi^{(1)}}{\sqrt{1-\lambda B_{0}}} \mathrm{~d} l, \\
\Upsilon=\frac{n^{\prime}}{n}+\frac{T^{\prime}}{T}\left(\frac{m v^{2}}{2 T}-\frac{3}{2}\right)+\frac{Z e \varphi_{0}^{\prime}}{T}
\end{gathered}
$$

and $C_{i i}^{\ell}$ is the linearized ion-ion collision operator (Helander \& Sigmar 2002). The ion-electron collision term has been neglected because we assume $\sqrt{m_{e} / m} \ll 1$, with $m_{e}$ the electron mass. The superindex $(0)$ in $C_{i i}^{\ell(0)}$ means that $B$ has been replaced by $B_{0}$ in the expression for the collision operator in coordinates $v$ and $\lambda$. Analogously,

$$
v_{\|}^{(0)}(r, \alpha, l, v, \lambda, \sigma)=\sigma v \sqrt{1-\lambda B_{0}(r, \alpha, l)},
$$

and $l_{b_{10}}, l_{b_{20}}$ are the solutions of the equation $1-\lambda B_{0}(r, \alpha, l)=0$ for $\lambda \geqslant B_{0, \text { max }}^{-1}$. Finally, note that $\partial_{r} J^{(0)}$ does not depend on $\alpha$ because $B_{0}$ is omnigenous. Sometimes, it will be useful to write $J^{(1)}=J_{B}^{(1)}+J_{\varphi}^{(1)}$, where

$$
J_{B}^{(1)}=-\lambda v \int_{l_{b_{10}}}^{l_{b_{20}}} \frac{B_{1}}{\sqrt{1-\lambda B_{0}}} \mathrm{~d} l
$$

and

$$
J_{\varphi}^{(1)}=-\frac{2 Z e}{m v} \int_{l_{b_{10}}}^{l_{b_{20}}} \frac{\varphi^{(1)}}{\sqrt{1-\lambda B_{0}}} \mathrm{~d} l .
$$

Second, one has the quasineutrality equation. In the mass ratio expansion $\sqrt{m_{e} / m}$ $\ll 1$, the non-adiabatic response of the electrons can be dropped, giving

$$
\left(\frac{Z}{T}+\frac{1}{T_{e}}\right) \varphi^{(1)}=\frac{2 \pi}{e n} \int_{0}^{\infty} \mathrm{d} v \int_{B_{0, \max }^{-1}}^{B_{0}^{-1}} \mathrm{~d} \lambda \frac{v^{3} B_{0}}{\left|v_{\|}^{(0)}\right|} g^{(1)},
$$

where $T_{e}(r)$ is the electron temperature. Note that the choice (2.17) implies that

$$
\left\langle\varphi^{(1)}\right\rangle=0
$$

where the flux-surface average is taken using $B_{0}$. In addition, integration of (2.25) over trapped orbits, application of property (2.11) and of condition (2.17) implies

$$
\int_{0}^{2 \pi} J_{\varphi}^{(1)} \mathrm{d} \alpha=0 .
$$

Equations (2.18) and (2.25) are correct for values of the collisionality that include the neoclassical transport regimes known as $1 / v, \sqrt{v}$ and superbanana-plateau regimes. In Calvo et al. (2017), it was explained why the equations stop to be valid at low enough values of the collisionality, so that they should be modified in case one is interested in describing, for example, the $v$ or superbanana regimes. In $\$ 2.4$, we explain how some of the quantities entering the neoclassical equations (2.18) and (2.25) behave when a large aspect ratio expansion is taken. In $\S 2.5$, the perturbed omnigenous magnetic field that will be later used in the numerical examples is described. 


\subsection{Conventions and some useful scalings for large aspect ratio stellarators}

Stellarators in operation today have large aspect ratios, $\epsilon^{-1}=R_{0} / a$. In Calvo et al. (2017), the scaling with $\epsilon \ll 1$ was not determined, and we would like to address this refinement in the present paper. In this subsection, some conventions that are convenient for $\epsilon \ll 1$ are explained, and the scaling with $\epsilon$ of several quantities entering (2.18) and (2.25) is provided for later use.

In a given flux surface, we write magnetic fields in large aspect ratio stellarators close to omnigeneity as

$$
B=B_{0}+\delta B_{1},
$$

where

$$
B_{0}=B_{00}+\tilde{B}_{0},
$$

$B_{00}$ is constant and $\tilde{B}_{0} \sim \epsilon B_{00}$. As for the non-omnigenous perturbation, we assume $B_{1} \sim \epsilon B_{00}$. Note that if the aspect ratio is large, the meaningful measure of the size of the non-omnigenous perturbation is given by $\delta \sim\left|B-B_{0}\right| /\left|B_{0}-B_{00}\right|$. This is why we have included a factor $\epsilon$ in our convention for the size of $B_{1}$. The definition

$$
\delta \sim \frac{B-B_{0}}{\epsilon B_{0}}
$$

is valid both for large aspect ratio, $\epsilon \ll 1$, and tight aspect ratio, $\epsilon \sim 1$.

The radial derivative of $B_{0}$ at the surface of interest will also be needed. In our models, we will assume

$$
\partial_{r} B_{0}=\frac{\tilde{B}_{0}}{\epsilon R_{0}} .
$$

We proceed to explain how different quantities appearing in the neoclassical equations scale with $\epsilon$. We denote by $\Delta_{\text {trapped }}$ the size (measured in the coordinate $\lambda$ ) of the region of phase space corresponding to particles trapped in $B_{0}$. Observing that the difference between the maximum and the minimum values of $B_{0}$ on the flux surface is $O\left(\epsilon B_{00}\right)$, we infer that

$$
\Delta_{\text {trapped }} \sim \epsilon B_{00}^{-1} .
$$

Analogous reasons lead to conclude that the size of the parallel velocity for trapped particles is

$$
v_{\|}^{(0)} \sim \epsilon^{1 / 2} v_{t} .
$$

The scaling of $\partial_{r} J^{(0)}$ depends on the size of the radial electric field, obtained by imposing ambipolarity of the neoclassical radial particle fluxes. For typical values $\varphi_{0}^{\prime} \sim$ $\epsilon^{-1} T /\left(e R_{0}\right)$, the term containing the radial electric field dominates and

$$
\partial_{r} J^{(0)} \sim \epsilon^{-3 / 2} v_{t},
$$

where we have assumed that the typical length of the omnigenous wells is $R_{0}$. If the radial electric field is small, $\left|\varphi_{0}^{\prime}\right| \lesssim T /\left(e R_{0}\right)$, then ${ }^{5}$

$$
\partial_{r} J^{(0)} \sim \epsilon^{-1 / 2} v_{t} .
$$

\footnotetext{
${ }^{5}$ See, for example, Klinger et al. (2017), where radial electric field profiles are shown such that $\varphi_{0}^{\prime}=0$ at some radial position.
} 
As for $J^{(1)}$, we have

$$
J_{B}^{(1)} \approx-B_{00}^{-1} v \int_{l_{b_{10}}}^{l_{b_{20}}} \frac{B_{1}}{\sqrt{1-\lambda B_{0}}} \mathrm{~d} l \sim \epsilon^{1 / 2} v_{t} R_{0},
$$

whereas

$$
J_{\varphi}^{(1)} \sim \epsilon^{-1 / 2} v_{t} R_{0} \frac{e \varphi^{(1)}}{T} .
$$

Recall that $\varphi^{(1)}$ is one of the unknowns of the neoclassical equations and its size is to be determined.

The function $\Psi$ appearing in (2.1) scales as

$$
\Psi \sim \epsilon B_{00} R_{0} .
$$

Regarding the plasma profile gradients, it is expected that, generically,

$$
\Upsilon \sim \frac{1}{\epsilon R_{0}}
$$

In large aspect ratio stellarators, the collision term on the right-hand side of (2.18) is dominated by the pitch-angle scattering piece of the collision operator,

$$
C_{i i}^{\ell(0)}\left[g^{(1)}\right] \approx v_{\lambda} \frac{v_{\|}^{(0)}}{v^{2} B_{00}^{2}} \partial_{\lambda}\left(v_{\|}^{(0)} \partial_{\lambda} g^{(1)}\right),
$$

where

$$
v_{\lambda}(v)=\frac{3 \sqrt{\pi}}{2 \tau_{i i}} \frac{\operatorname{erf}(v \sqrt{m /(2 T)})-\chi(v \sqrt{m /(2 T)})}{(v \sqrt{m /(2 T)})^{3}}
$$

is the ion-ion collision frequency,

$$
\tau_{i i}=6 \sqrt{2} \pi^{3 / 2} \varepsilon_{0}^{2} m^{1 / 2} T^{3 / 2} /\left(Z^{4} e^{4} n \ln \Lambda\right),
$$

$\ln \Lambda$ is the Coulomb logarithm, $\varepsilon_{0}$ is the vacuum permittivity,

$$
\operatorname{erf}(x)=\frac{2}{\sqrt{\pi}} \int_{0}^{x} e^{-y^{2}} \mathrm{~d} y
$$

and

$$
\chi(x)=\frac{\operatorname{erf}(x)-(2 x / \sqrt{\pi}) \exp \left(-x^{2}\right)}{2 x^{2}} .
$$

Employing the estimates above for the scaling of $\Delta_{\text {trapped }}$ and $v_{\|}^{(0)}$ with $\epsilon$, we find

$$
C_{i i}^{\ell(0)}\left[g^{(1)}\right] \sim \frac{v_{*}}{\epsilon} \frac{v_{t}}{R_{0}} g^{(1)},
$$

which is the typical size of the collision term across the trapped region. It is important to realize that the condition for low collisionality ions, that reads $\nu_{*} \ll 1$ for $\epsilon \sim 1$, becomes $^{6} \nu_{*} \ll \epsilon^{3 / 2}$ for $\epsilon \ll 1$. This result is derived by comparing the factor in front of

${ }^{6}$ We point out that the range $\epsilon^{3 / 2} \ll \nu_{*} \ll 1$ corresponds to the plateau regime. 
$g^{(1)}$ on the right-hand side of (2.45) with the typical frequency of the parallel particle motion, which is $O\left(\epsilon^{1 / 2} v_{t} / R_{0}\right)$ when $\epsilon \ll 1$.

Finally, we point out that (see (2.40)), when $\epsilon \ll 1$, we can define

$$
v_{*}:=\frac{v_{\lambda}\left(v_{t}\right) R_{0}}{v_{t}}
$$

as the expression for the collisionality and

$$
\rho_{*}:=\frac{\sqrt{m T}}{Z e B_{00} R_{0}}
$$

for the normalized Larmor radius.

\subsection{Model magnetic field close to omnigeneity}

In order to define a specific omnigenous field $B_{0}$ for the numerical examples provided in subsequent sections, we follow Cary \& Shasharina (1997) and Landreman \& Catto (2012). The description is easier in Boozer coordinates (Boozer 1981). We denote by $\theta$ and $\zeta$ the poloidal and toroidal Boozer angles, respectively. We use the construction explained in section V of Landreman \& Catto (2012) with $M=1$ and $N=4$ (which means that the contours of $B_{0}$ close on themselves after 4 poloidal turns and 1 toroidal turn) and with rotational transform $t=1.05$. We take ${ }^{7}$

$$
\tilde{B}_{0}(\theta, \zeta)=\epsilon B_{00} \cos \eta(\theta, \zeta),
$$

where the function $\eta$ is obtained as in equation (73) of Landreman \& Catto (2012). In figure 2 we show a plot of an omnigenous magnetic field of the form just introduced.

As for the non-omnigenous perturbation, we take

$$
B_{1}=\epsilon B_{00} \cos (2 \theta) .
$$

Note that $B=B_{0}+\delta B_{1}$ is symmetric under the transformation $(\theta, \zeta) \mapsto(-\theta,-\zeta)$. Magnetic fields with this property are called stellarator symmetric (Dewar \& Hudson 1998). Stellarator designs typically impose this symmetry on the magnetic configuration, and from here on we always assume that our magnetic field satisfies this.

Given Boozer angles $\{\theta, \zeta\}$, there are many different ways to define coordinates $\{\alpha, l\}$. First, one has to choose a closed curve $\mathcal{C}$ that is not contractible to a point. A simple choice is to take the curve $\zeta=0$. Then,

$$
\alpha:=\theta-t \zeta .
$$

As for the arc length $l$,

$$
l(\alpha, \zeta)=\int_{0}^{\zeta} \frac{1}{\hat{\boldsymbol{b}} \cdot \nabla \zeta^{\prime}} \mathrm{d} \zeta^{\prime},
$$

where the integral on the right-hand side is taken at constant $\alpha$ and $l_{\max }$ is determined by the intersection of the field line with $\mathcal{C}$ when it goes all the way around the torus.

\footnotetext{
${ }^{7}$ In order to avoid any confusion, we point out that the meaning of the symbol $\epsilon$ in our paper and in Landreman \& Catto (2012) is not exactly the same. What we call $\epsilon$ would be called $\epsilon /(1+\epsilon)$ in Landreman \& Catto (2012). What we call $B_{00}(1-\epsilon)$ would be called $\breve{B}$ in Landreman \& Catto (2012).
} 


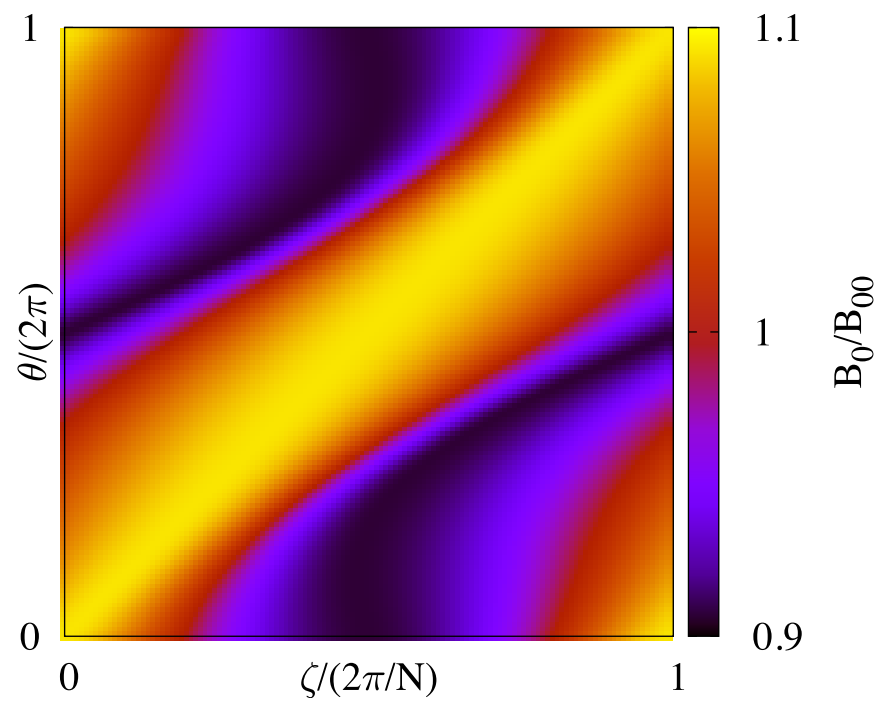

FIGURE 2. Plot of $B_{0}$ for the model omnigenous magnetic field described at the beginning of $\S 2.5$; that is, $B_{0}=B_{00}+\tilde{B}_{0}$, with $\tilde{B}_{0}$ of the form (2.48). Here, $\epsilon=0.1$.

\section{Dependence of $\varphi^{(1)}$ on the collisionality and the radial electric field: a realistic numerical example}

Let us take realistic, stellarator reactor-relevant parameters, $\rho_{*}=1.87 \times 10^{-4}$ and $\epsilon=0.1$, and let us employ the model magnetic field close to omnigeneity $B=B_{0}+\delta B_{1}$ described in $\$ 2.5$ (see (2.29), (2.48) and (2.49)). As for the profiles, we take $n^{\prime} / n=$ $-\left(\epsilon R_{0}\right)^{-1}$ and $T^{\prime}=0$. We solve (2.18) and (2.25) with the code KNOSOS, and in figure 3 we represent the size of $\varphi^{(1)}$ versus $v_{*}$. We plot curves corresponding to $\varphi_{0}^{\prime}=0$ and to $\varphi_{0}^{\prime}=2 \epsilon^{-1} T /\left(e R_{0}\right)$. In this plot and in subsequent ones representing $\varphi^{(1)}$ versus $\nu_{*}$, we typically take the maximum value of $\varphi^{(1)}$ on the flux surface, and this is what we generally understand by 'the size of $\varphi^{(1)}$. However, there are regimes (see $\S 4.3$ ) in which the scaling of $\varphi^{(1)}$ at special points (lying in a very small spatial region associated with certain resonant trajectories) is different from that of $\varphi^{(1)}$ at generic points, far from the special points. When such regimes exist, we usually plot two curves and distinguish between 'the size of $\varphi^{(1)}$, (see, for example, empty squares in figure 3), given by the maximum of $\varphi^{(1)}$ on the flux surface, and the size of $\varphi^{(1)}$ at generic points' (see, for example, full squares in figure 3). In order to compute the size of $\varphi^{(1)}$ at generic points, we take a spatial average of $\varphi^{(1)}$ in a region where $\varphi^{(1)}$ is positive and which is far from the special points.

The dependence of $\varphi^{(1)}$ on the collisionality and on the radial electric field shown in figure 3 is non-trivial. Understanding the different regimes encoded in a plot like figure 3 is the main subject of this paper. We will identify and study these regimes in $\S 4$. As we will explain below, in order to have very clear numerical scalings in all the asymptotic regimes that (2.18) and (2.25) admit, in $\S 4$ we will employ extremely small values of $\rho_{*}$ and $\epsilon$ in our numerical checks. The reader that prefers to skip the mathematical derivations of $\S 4$ can go directly to table 1 in $\S 5$ for a collection of the main analytical results. 


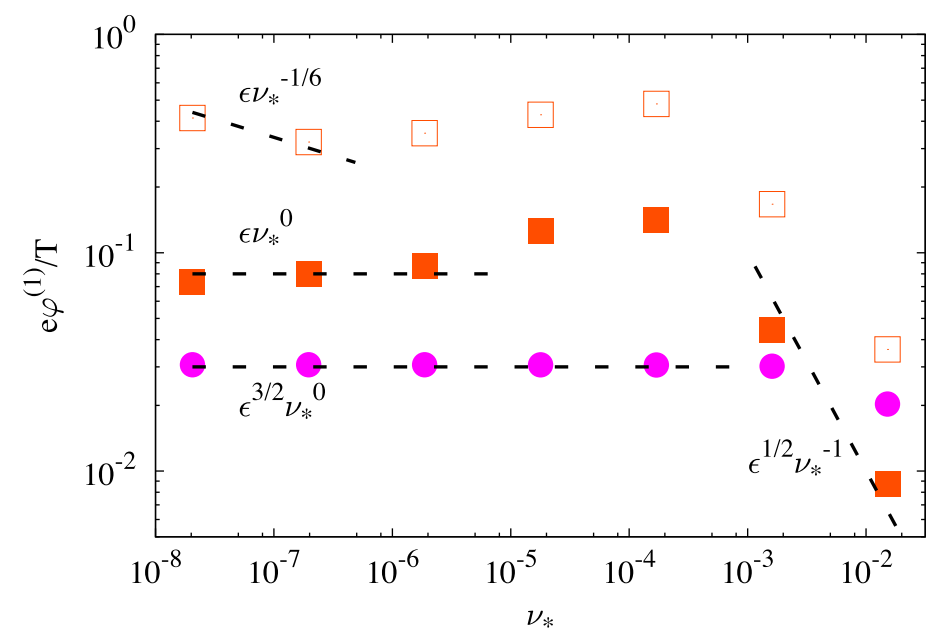

FIGURE 3. Size of $\varphi^{(1)}$ as a function of the collisionality for a plasma with $\rho_{*}=1.87 \times$ $10^{-4}$ and magnetic configuration defined at the end of $\S 2.5$ with $\epsilon=0.1$. The circles correspond to results with $\varphi_{0}^{\prime}=2 \epsilon^{-1} T /\left(e R_{0}\right)$. The squares correspond to $\varphi_{0}^{\prime}=0$. Empty squares give the size of $\varphi^{(1)}$. Full squares give the size of $\varphi^{(1)}$ at generic points (see the main text for the explanation on the difference between size of $\varphi^{(1)}$ and size of $\varphi^{(1)}$ at generic points). The important difference between the curves consisting of empty and full squares is that, for $v_{*} \ll \rho_{*}$, the first one behaves as $v_{*}^{-1 / 6}$ and the second one does not vary with $v_{*}$.

\section{Asymptotic regimes of $\varphi^{(1)}$ at low collisionality}

In this section, we discuss the solution $\varphi^{(1)}$ determined by equations (2.18) and (2.25) in three asymptotic low collisionality regimes. Namely, the $1 / v$ regime, the $\sqrt{v}$ regime and the superbanana-plateau regime (the names of the regimes are due to the scaling of the radial neoclassical fluxes with collisionality, not necessarily to the scaling of $\varphi^{(1)}$, as we will explain below). The subsection devoted to each regime will start with a discussion valid for arbitrary aspect ratio. Then, the results will be particularized for large aspect ratio stellarators and numerical examples of the most relevant analytical results will follow. In the course of the analytical derivations, it will become clear that, in order to clearly distinguish all the asymptotic regimes that equations (2.18) and (2.25) admit, the quantities $\rho_{*}, \rho_{*} / \epsilon^{1 / 2}$ and $\rho_{*} / \epsilon$ need to be sufficiently separated from each other. This is why, in what follows, we will take artificially small values for $\rho_{*}$ and $\epsilon$, so that there is enough room between those quantities in our numerical examples. In these examples, we will always take $\rho_{*}=$ $1.1 \times 10^{-12}, n^{\prime} / n=-\left(\epsilon R_{0}\right)^{-1}$ and $T^{\prime}=0$, and we will always employ the model magnetic field close to omnigeneity defined in $\$ 2.5$ (recall equations (2.29), (2.48) and (2.49)). We will use different values of $\epsilon$ and $\varphi_{0}^{\prime}$ that will be specified where appropriate.

\subsection{The $1 / v$ regime}

For tight aspect ratio stellarators, the $1 / v$ regime corresponds to $\rho_{*} \ll v_{*} \ll 1$. In this situation, the first term on the left-hand side of (2.18) is negligible compared to the 
term on the right-hand side,

$$
\left|\partial_{r} J^{(0)} \partial_{\alpha} g^{(1)}\right| \ll\left|\sum_{\sigma} \frac{Z e \Psi}{m} \int_{l_{b_{10}}}^{l_{b_{20}}} \frac{\mathrm{d} l}{\left|v_{\|}^{(0)}\right|} C_{i i}^{\ell(0)}\left[g^{(1)}\right]\right| .
$$

The correction to the Maxwellian distribution in the $1 / v$ regime is therefore found by integrating the expression

$$
\sum_{\sigma} \frac{Z e \Psi}{m} \int_{l_{b_{10}}}^{l_{b_{20}}}\left|v_{\|}^{(0)}\right|^{-1} C_{i i}^{\ell(0)}\left[g_{1 / \nu}^{(1)}\right] \mathrm{d} l=\partial_{\alpha} J^{(1)} \Upsilon F_{M} .
$$

Below, we will find useful to distinguish between $g_{1 / \nu}^{(1)}\left[J_{B}^{(1)}\right]$ and $g_{1 / \nu}^{(1)}\left[J_{\varphi}^{(1)}\right]$, where

$$
\sum_{\sigma} \frac{Z e \Psi}{m} \int_{l_{b_{10}}}^{l_{b_{20}}}\left|v_{\|}^{(0)}\right|^{-1} C_{i i}^{\ell(0)}\left[g_{1 / \nu}^{(1)}\left[J_{B}^{(1)}\right]\right] \mathrm{d} l=\partial_{\alpha} J_{B}^{(1)} \Upsilon F_{M}
$$

and

$$
\sum_{\sigma} \frac{Z e \Psi}{m} \int_{l_{b_{10}}}^{l_{b_{20}}}\left|v_{\|}^{(0)}\right|^{-1} C_{i i}^{\ell(0)}\left[g_{1 / \nu}^{(1)}\left[J_{\varphi}^{(1)}\right]\right] \mathrm{d} l=\partial_{\alpha} J_{\varphi}^{(1)} \Upsilon F_{M}
$$

and therefore $g_{1 / \nu}^{(1)}=g_{1 / \nu}^{(1)}\left[J_{B}^{(1)}\right]+g_{1 / \nu}^{(1)}\left[J_{\varphi}^{(1)}\right]$. We note that

$$
g_{1 / \nu}^{(1)}\left[J_{B}^{(1)}\right] \sim \frac{\rho_{*}}{v_{*}} \frac{n}{v_{t}^{3}}
$$

and

$$
g_{1 / \nu}^{(1)}\left[J_{\varphi}^{(1)}\right] \sim \frac{\rho_{*}}{v_{*}} \frac{e \varphi^{(1)}}{T} \frac{n}{v_{t}^{3}} .
$$

The equation for $\varphi^{(1)}$ reads (recall (2.25))

$$
\varphi^{(1)}=\left(\frac{Z}{T}+\frac{1}{T_{e}}\right)^{-1} \frac{2 \pi B_{0}}{e n} \int_{0}^{\infty} \mathrm{d} v v^{3} \int_{B_{0, \max }^{-1}}^{B_{0}^{-1}} \mathrm{~d} \lambda\left|v_{\|}^{(0)}\right|^{-1}\left(g_{1 / \nu}^{(1)}\left[J_{\varphi}^{(1)}\right]+g_{1 / \nu}^{(1)}\left[J_{B}^{(1)}\right]\right) .
$$

Using (4.6) and $\rho_{*} \ll v_{*}$, and comparing the size of the left-hand side of (4.7) with the size of the term on the right-hand side containing $g_{1 / \nu}^{(1)}\left[J_{\varphi}^{(1)}\right]$, we conclude that the latter can be dropped. Hence, $\varphi^{(1)}$ is calculated as

$$
\varphi^{(1)}=\left(\frac{Z}{T}+\frac{1}{T_{e}}\right)^{-1} \frac{2 \pi B_{0}}{e n} \int_{0}^{\infty} \mathrm{d} v v^{3} \int_{B_{0, \max }^{-1}}^{B_{0}^{-1}} \mathrm{~d} \lambda\left|v_{\|}^{(0)}\right|^{-1} g_{1 / v}^{(1)}\left[J_{B}^{(1)}\right] .
$$

Then, in the $1 / v$ regime (see (4.5) and (4.6)),

$$
g^{(1)} \sim \frac{\rho_{*}}{v_{*}} \frac{n}{v_{t}^{3}}
$$

and

$$
\varphi^{(1)} \sim \frac{\rho_{*}}{v_{*}} \frac{T}{e} .
$$

If $B_{0}$ and $B_{1}$ are stellarator symmetric, $J_{B}^{(1)}$ is symmetric and, consequently, $\partial_{\alpha} J_{B}^{(1)}$ is antisymmetric. Since the collision operator does not change the symmetry, the solution $g_{1 / \nu}^{(1)}$ of (4.2) is antisymmetric. Finally, $\varphi^{(1)}$, obtained from (4.8), is stellarator antisymmetric. 


\subsubsection{The $1 / v$ regime in a large aspect ratio stellarator}

As indicated by the end of $\S 2.4$, when $\epsilon$ is small the low collisionality condition reads $\nu_{*} \ll \epsilon^{3 / 2}$. How small $\nu_{*}$ can be before the tangential drifts start to count depends on their size, as will be discussed mainly in $\S \S 4.2 .1$ and 4.2.2.

Let us refine the estimates (4.9) and (4.10) for the distribution function and the electrostatic potential when $\epsilon \ll 1$. The drift-kinetic equation for $\epsilon \ll 1$ is

$$
\frac{2 Z e \Psi}{m B_{00}^{2}} \partial_{\lambda}\left[\left(\int_{b_{10}}^{l_{20}} \sqrt{1-\lambda B_{0}} \mathrm{~d} l\right) \partial_{\lambda} g_{1 / v}^{(1)}\right]=\frac{v}{v_{\lambda}} \partial_{\alpha} J^{(1)} \Upsilon F_{M} .
$$

Employing the scalings of $\S 2.4$, it is easy to infer that

$$
g_{1 / v}^{(1)}\left[J_{B}^{(1)}\right] \sim \frac{\rho_{*}}{v_{*}} \frac{n}{v_{t}^{3}}
$$

and

$$
g_{1 / \nu}^{(1)}\left[J_{\varphi}^{(1)}\right] \sim \epsilon^{-1 / 2} \frac{\rho_{*}}{v_{*}} \frac{J_{\varphi}^{(1)}}{v_{t} R_{0}} \frac{n}{v_{t}^{3}} .
$$
If

Next, we apply the $\epsilon \ll 1$ expansion to (4.7). We must treat two cases separately.

$$
\epsilon^{-1 / 2} \rho_{*} \ll v_{*} \ll \epsilon^{3 / 2},
$$

then the first term on the right-hand side of (4.7) is negligible with respect to the left-hand side. Using that $\Delta_{\text {trapped }} \sim \epsilon B_{00}^{-1}$, we find

$$
\varphi^{(1)} \sim \epsilon^{1 / 2} \frac{\rho_{*}}{v_{*}} \frac{T}{e} .
$$

And noting that $g_{1 / \nu}^{(1)}\left[J_{\varphi}^{(1)}\right] \ll g_{1 / v}^{(1)}\left[J_{B}^{(1)}\right]$,

$$
g^{(1)} \sim \frac{\rho_{*}}{v_{*}} \frac{n}{v_{t}^{3}} .
$$

Then, both $\varphi^{(1)}$ and $g^{(1)}$ scale as $v_{*}^{-1}$. The fact that $\varphi^{(1)}$ is antisymmetric is also clear. In figure 4 , a contour plot of $\varphi^{(1)^{*}}$ on the magnetic surface is provided for a set of values such that (4.14) holds.

However, if $v_{*} \sim \epsilon^{-1 / 2} \rho_{*}$ or smaller, the first term on the right-hand side of (4.7) can become comparable to the left-hand side. Let us study the asymptotic regime

$$
\nu_{*} \ll \epsilon^{-1 / 2} \rho_{*} .
$$

From (4.7) we learn that $\varphi^{(1)}$ depends on $l$ only through $B_{0}$. This will be important in a moment. When (4.17) holds, the left-hand side of (4.7) is small compared to the term on the right-hand side that involves $\varphi^{(1)}$. Then, we can write

$$
\int_{0}^{\infty} \mathrm{d} v v^{2} \int_{B_{0, \max }^{-1}}^{B_{0}^{-1}} \mathrm{~d} \lambda \frac{1}{\sqrt{1-\lambda B_{0}}} g_{1 / v}^{(1)}=0
$$

where $g_{1 / v}^{(1)}$ is the solution of (4.11). For the argument that follows, we find it convenient to write $J^{(1)}$ as

$$
J^{(1)}=-\int_{B_{0, \min }}^{B_{0}} \frac{G}{\sqrt{1-\lambda \hat{B}}} \mathrm{~d} \hat{B} .
$$




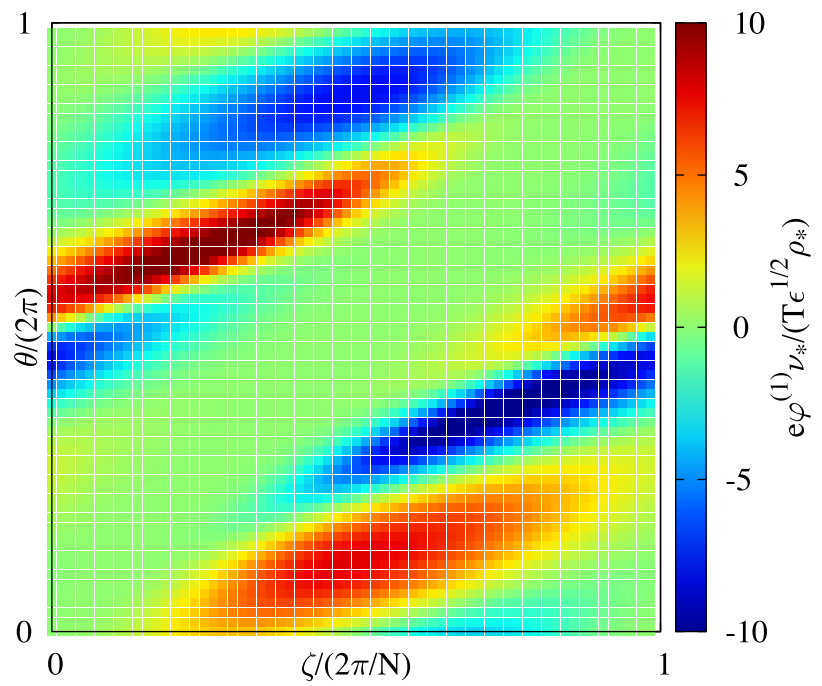

FIgURE 4. Contour plot of $\varphi^{(1)}$ for $\epsilon=1.1 \times 10^{-4}, v_{*}=10^{-7}$ and $\varphi_{0}^{\prime}=0$.

Here, $B_{0, \min }$ is the minimum of $B_{0}$,

$$
G(r, \alpha, \hat{B}, \beta, v):=\sum_{\beta=-1}^{1}\left(\frac{B_{1} v}{B_{00}}+\frac{2 Z e \varphi^{(1)}}{m v}\right) \frac{1}{\left|\partial_{l} B_{0}\right|}
$$

and we have used the magnitude of $B_{0}$ as the integration variable in (4.19). Since there are two values ${ }^{8}$ of $l$ corresponding to each value $B_{0}=\hat{B}$, we introduce the discrete coordinate $\beta= \pm 1$. The value $\beta=-1$ labels the branch of the magnetic well where $\partial_{l} B_{0}<0$ and the value $\beta=1$ labels the branch where $\partial_{l} B_{0} \geqslant 0$. On the right-hand side of (4.20), every function is understood to be expressed using $\hat{B}$ and $\beta$ instead of $l$. In particular, we note that, in general, $\left|\partial_{l} B_{0}\right|(\hat{B}, \beta) \neq\left|\partial_{l} B_{0}\right|(\hat{B},-\beta)$.

It is clear that

$$
\int_{0}^{\infty} v^{2} g_{1 / \nu}^{(1)} \mathrm{d} v=0
$$

gives the solution of (4.18) and that, by imposing (4.21), we obtain a relation between the orbit integrals of $B_{1}$ and $\varphi^{(1)}$. The orbit integral of a function can be interpreted as a transformation that replaces the coordinate $l$ by the coordinate $\lambda$. This can be viewed as an Abel transform, and an explicit inversion formula is given in appendix A. In principle, the inversion cannot distinguish between two points of the magnetic well with the same value of $B_{0}$ (this is quite intuitive when we use the magnitude of $B_{0}$ as the integration variable, as we have done in (4.19)). Carrying out the integral in $v$ on the left-hand side of (4.21), we arrive at

$$
\sum_{\beta=-1}^{1} \frac{Z e \varphi^{(1)}}{T} \frac{1}{\left|\partial_{l} B_{0}\right|}=-2.83 \frac{n^{\prime} / n+Z e \varphi_{0}^{\prime} / T+2.33 T^{\prime} / T}{n^{\prime} / n+Z e \varphi_{0}^{\prime} / T+1.33 T^{\prime} / T} \sum_{\beta=-1}^{1} \frac{B_{1}}{B_{00}} \frac{1}{\left|\partial_{l} B_{0}\right|} .
$$

${ }^{8}$ For simplicity in the presentation, we assume that the omnigenous wells are such that exactly two points in the well take each value $B_{0}=\hat{B}$ (except for the value $B_{0, \min }$, which is reached at a single point). There exist omnigenous magnetic fields with more complicated wells (Parra et al. 2015), and in those cases the discrete coordinate $\beta$ would need to label more than two branches. 


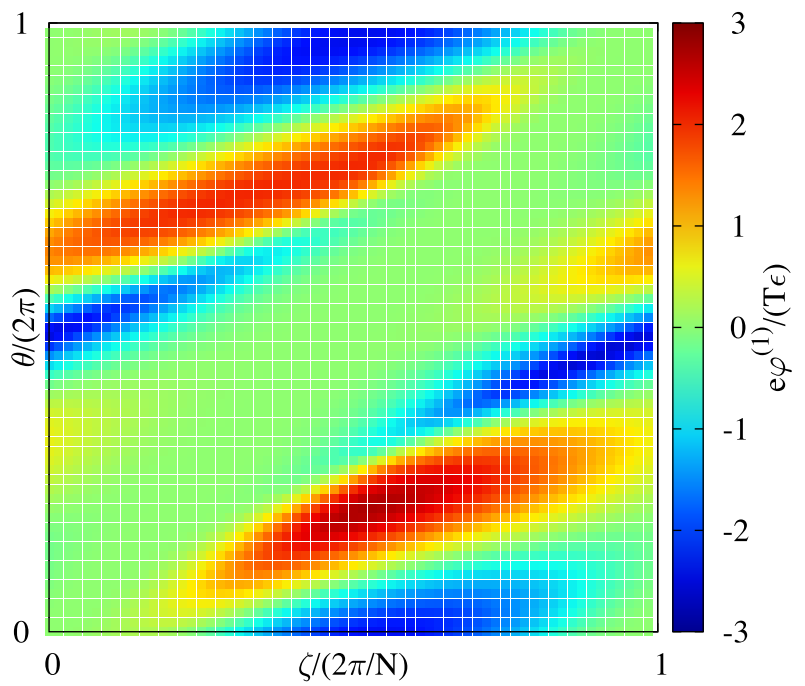

FIgURE 5. Contour plot of $\varphi^{(1)}$ for $\epsilon=1.1 \times 10^{-4}, v_{*}=5.8 \times 10^{-11}$ and $\varphi_{0}^{\prime}=0$.

Recalling that $\varphi^{(1)}$ depends on $l$ only through $B_{0}$ (see (4.7)), we finally obtain the explicit solution

$$
\frac{Z e \varphi^{(1)}}{T}=-2.83 \frac{n^{\prime} / n+Z e \varphi_{0}^{\prime} / T+2.33 T^{\prime} / T}{n^{\prime} / n+Z e \varphi_{0}^{\prime} / T+1.33 T^{\prime} / T} \frac{\sum_{\beta=-1}^{1}\left(B_{1} / B_{00}\right)\left|\partial_{l} B_{0}\right|^{-1}}{\sum_{\beta=-1}^{1}\left|\partial_{l} B_{0}\right|^{-1}}
$$

From (4.23) we infer that in this regime $\varphi^{(1)}$ is stellarator symmetric and that its size, in general, is

$$
\varphi^{(1)} \sim \epsilon \frac{T}{e}
$$

We note that for particular combinations of the profile gradients, $\varphi^{(1)}$ can get larger because the denominator of (4.23) can become small. This will be studied elsewhere.

In figure 5, a contour plot of $\varphi^{(1)}$ on the magnetic surface is provided for a set of values that satisfy (4.17).

Finally, we have (see (4.12) and (4.13))

$$
g_{1 / \nu}^{(1)} \sim g_{1 / \nu}^{(1)}\left[J_{\varphi}^{(1)}\right] \sim g_{1 / \nu}^{(1)}\left[J_{B}^{(1)}\right] \sim \frac{\rho_{*}}{\nu_{*}} \frac{n}{v_{t}^{3}} .
$$

In figure 6 , and for $\varphi_{0}^{\prime}=0$, we illustrate the scaling of $\varphi^{(1)}$ and of

$$
n_{B}:=\int g_{1 / \nu}^{(1)}\left[J_{B}^{(1)}\right] \mathrm{d}^{3} v \sim \frac{\rho_{*}}{\mathcal{V}_{*}} n
$$

in a range of collisionality values such that both regimes, (4.14) and (4.17), are observed. Whereas $\varphi^{(1)}$ and $n_{B}$ have the same scaling with $v_{*}$ in the regime (4.14), they scale differently in the regime (4.17). 


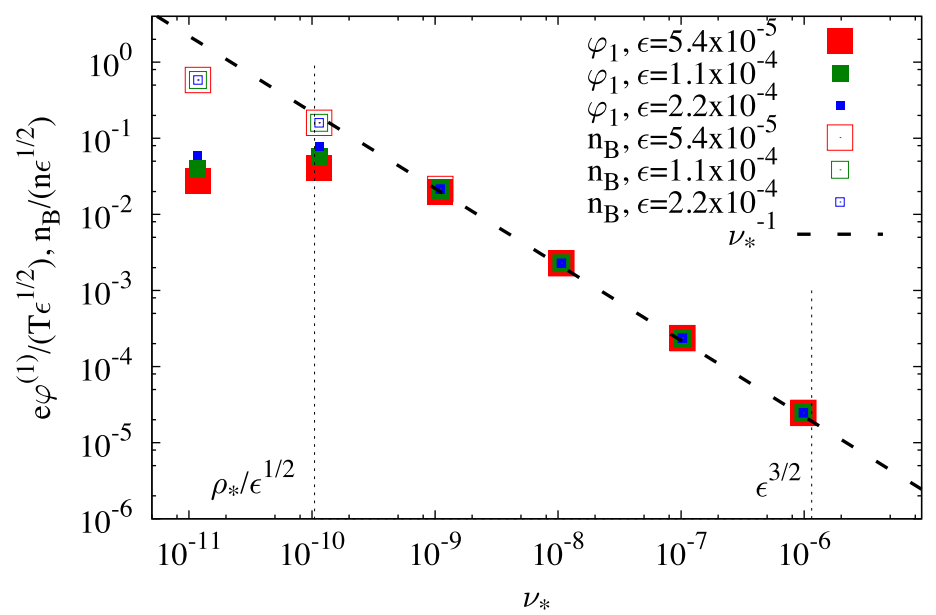

FIgURE 6. Scaling of $\varphi^{(1)}$ and $n_{B}$ (evaluated at the point of the flux surface where they reach their maximum values) with $\nu_{*}$ and $\epsilon$. Here, $\varphi_{0}^{\prime}=0$. The regimes (4.14) and (4.17) are confirmed numerically. The central value $\epsilon=1.1 \times 10^{-4}$ has been used to draw the thin dotted lines indicating where the collisionality values $\rho_{*} / \epsilon^{1 / 2}$ and $\epsilon^{3 / 2}$ are located.

\subsection{The $\sqrt{v}$ regime}

We turn to discuss collisionality regimes with $v_{*} \ll \rho_{*} \ll 1$. In this situation, as explained at length in Calvo et al. (2017), the behaviour of both the radial neoclassical fluxes and $\varphi^{(1)}$ depends on the zeroes of $\partial_{r} J^{(0)}$.

When $v_{*} \ll \rho_{*}$, the collision term in (2.18) is negligible compared to the first term on the left-hand side,

$$
\left|\sum_{\sigma} \frac{Z e \Psi}{m} \int_{l_{b_{10}}}^{l_{b_{20}}} \frac{\mathrm{d} l}{\left|v_{\|}^{(0)}\right|} C_{i i}^{\ell(0)}\left[g^{(1)}\right]\right| \ll\left|\partial_{r} J^{(0)} \partial_{\alpha} g^{(1)}\right| .
$$

Hence, to lowest order in an expansion in $\nu_{*} / \rho_{*}$,

$$
g^{(1)}=g_{0}+\cdots,
$$

where $g_{0}$ is the solution of

$$
-\partial_{r} J^{(0)} \partial_{\alpha} g_{0}+\partial_{\alpha} J^{(1)} \Upsilon F_{M}=0
$$

This equation can be readily solved, obtaining an explicit expression for $g_{0}$,

$$
g_{0}=\frac{1}{\partial_{r} J^{(0)}}\left(J^{(1)}-\frac{1}{2 \pi} \int_{0}^{2 \pi} J^{(1)} \mathrm{d} \alpha\right) \Upsilon F_{M},
$$

where we have chosen $\int_{0}^{2 \pi} g_{0} \mathrm{~d} \alpha=0$ to fix the integration constant. From (4.30), it is clear that the solution $g_{0}$ is not valid at phase space points where $\partial_{r} J^{(0)}$ vanishes, and we devote $\S 4.3$ to those points. However, if $\partial_{r} J^{(0)}$ does not vanish at any point of phase space (or if it vanishes only for values of the velocity $v \gg v_{t}$, that are irrelevant 
because their effect is supressed by the smallness of the Maxwellian distribution), (4.30) is enough to correctly determine $\varphi^{(1)}$ via the quasineutrality equation

$$
\varphi^{(1)}=\left(\frac{Z}{T}+\frac{1}{T_{e}}\right)^{-1} \frac{2 \pi B_{0}}{e n} \int_{0}^{\infty} \mathrm{d} v v^{3} \int_{B_{0, \max }^{-1}}^{B_{0}^{-1}} \mathrm{~d} \lambda\left|v_{\|}^{(0)}\right|^{-1} g_{0} .
$$

In this case, $\varphi^{(1)}$ is stellarator symmetric (due to the fact that the expression (4.30) for $g_{0}$ does not involve derivatives of $B$ along the flux surface) and does not scale with collisionality,

$$
\varphi^{(1)} \sim \frac{T}{e} .
$$

As proven in Calvo et al. (2017), $g_{0}$ does not contribute to the radial neoclassical fluxes. They are determined by corrections to $g_{0}$ in (4.28) that are localized in a region of phase space that has a typical size $\Delta_{\sqrt{v}} \sim \sqrt{\nu_{*} / \rho_{*}}$ in the coordinate $\lambda$. This small layer produces the scaling with collisionality that justifies the name of this regime, the $\sqrt{v}$ regime.

If the aspect ratio is large, it is interesting to distinguish two cases (mostly in connection to the solutions of the quasineutrality equation), to which we devote $\S \S 4.2 .1$ and 4.2.2.

\subsubsection{Large aspect ratio and large tangential drift, $\partial_{r} J^{(0)} \sim \epsilon^{-3 / 2} v_{t} R_{0}$}

If $\partial_{r} J^{(0)} \sim \epsilon^{-3 / 2} v_{t} R_{0}$, the inequality (4.27) holds provided that $\nu_{*} \ll \epsilon^{-1} \rho_{*}$. Hence, the $1 / v$ regime takes place in the range

$$
\epsilon^{-1} \rho_{*} \ll v_{*} \ll \epsilon^{3 / 2} .
$$

In particular, the regime defined by (4.17) is not observed because the tangential drift starts to count for collisionalities much larger than $\epsilon^{-1 / 2} \rho_{*}$. It can be observed, however, when the tangential drift is smaller (see $\S 4.2 .2$ ).

As pointed out in $\S 2.4, \partial_{r} J^{(0)} \sim \epsilon^{-3 / 2} v_{t} R_{0}$ corresponds to $\varphi_{0}^{\prime} \sim \epsilon^{-1} T /\left(e R_{0}\right)$, which is the standard size of the radial electric field in a large aspect ratio stellarator. The derivation of the radial fluxes in this regime can be found, for example, in Galeev \& Sagdeev (1979), Ho \& Kulsrud (1987).

Let us start the discussion about $\varphi^{(1)}$ by writing (4.31) more explicitly. Inserting in (4.31) the expression for $g_{0}$ (see (4.30)) and rearranging, we get

$$
\begin{aligned}
\varphi^{(1)} & -\frac{2 \pi}{e n}\left(\frac{Z}{T}+\frac{1}{T_{e}}\right)^{-1} \int_{0}^{\infty} \mathrm{d} v \int_{B_{0, \max }^{-1}}^{B_{0}^{-1}} \mathrm{~d} \lambda \frac{v^{3} B_{0}}{\left|v_{\|}^{(0)}\right|} \frac{\Upsilon F_{M}}{\partial_{r} J^{(0)}} \widetilde{J}_{\varphi}^{(1)} \\
& =\frac{2 \pi}{e n}\left(\frac{Z}{T}+\frac{1}{T_{e}}\right)^{-1} \int_{0}^{\infty} \mathrm{d} v \int_{B_{0, \max }^{-1}}^{B_{0}^{-1}} \mathrm{~d} \lambda \frac{v^{3} B_{0}}{\left|v_{\|}^{(0)}\right|} \frac{\Upsilon F_{M}}{\partial_{r} J^{(0)}} \widetilde{J}_{B}^{(1)},
\end{aligned}
$$

where

$$
\widetilde{J}_{B}^{(1)}=J_{B}^{(1)}-\frac{1}{2 \pi} \int_{0}^{2 \pi} J_{B}^{(1)} \mathrm{d} \alpha
$$

and

$$
\widetilde{J}_{\varphi}^{(1)}=J_{\varphi}^{(1)}-\frac{1}{2 \pi} \int_{0}^{2 \pi} J_{\varphi}^{(1)} \mathrm{d} \alpha
$$




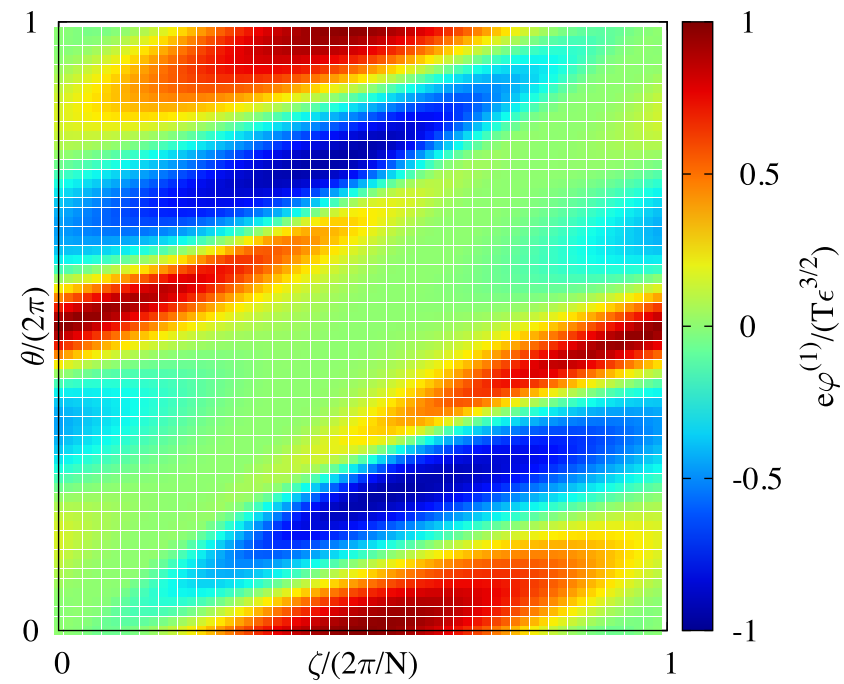

FIGURE 7. Contour plot of $\varphi^{(1)}$ for $\epsilon=1.1 \times 10^{-4}, \nu_{*}=1.1 \times 10^{-10}$ and $\varphi_{0}^{\prime}=2 \epsilon^{-1} T /\left(e R_{0}\right)$.

In (4.36) we have emphasized that only the component of $J_{\varphi}^{(1)}$ that fluctuates in $\alpha$ enters (4.34) although, strictly speaking, it is superfluous due to (2.27).

Recalling the estimates in $\S 2.4$, it is straightforward to see that the second term on the left-hand side of (4.34) is smaller than the first one by a factor $\epsilon^{1 / 2}$. Hence, the size of $\varphi^{(1)}$ is determined by the size of the right-hand side of (4.34). That is,

$$
\varphi^{(1)} \sim \epsilon^{3 / 2} \frac{T}{e} .
$$

This scaling is consistent with (4.15) when $v_{*} \sim \epsilon^{-1} \rho_{*}$. In figure 7, a contour plot of $\varphi^{(1)}$ corresponding to $\partial_{r} J^{(0)} \sim \epsilon^{-3 / 2} v_{t} R_{0}$ and $\nu_{*} \ll \epsilon^{-1} \rho_{*}$ is shown. In figure 8 , the collisionality-independent scaling (4.37) for $\varphi^{(1)}$ is numerically checked.

4.2.2. Large aspect ratio and small tangential drift, $\partial_{r} J^{(0)} \sim \epsilon^{-1 / 2} v_{t} R_{0}$

If $\partial_{r} J^{(0)} \sim \epsilon^{-1 / 2} v_{t} R_{0}$, (4.27) is satisfied when $v_{*} \ll \rho_{*}$. Therefore, for such typical size of the tangential drifts, the $1 / v$ regime happens for collisionality values

$$
\rho_{*} \ll \nu_{*} \ll \epsilon^{3 / 2} .
$$

To be precise, in the range $\rho_{*} \ll v_{*} \ll \epsilon^{-1 / 2} \rho_{*}$, the subregime (4.17) of the $1 / v$ regime will be observed.

When $\partial_{r} J^{(0)} \sim \epsilon^{-1 / 2} v_{t} R_{0}$, the left-hand side of (4.31) can be neglected compared to the term on the right-hand side containing $\varphi^{(1)}$, so that we have

$$
\int_{0}^{\infty} \mathrm{d} v v^{2} \int_{B_{0, \max }^{-1}}^{B_{0}^{-1}} \mathrm{~d} \lambda \frac{g_{0}}{\sqrt{1-\lambda B_{0}}}=0,
$$

which can be solved by finding the solution of

$$
\int_{0}^{\infty} \mathrm{d} v v^{2} g_{0}=0 .
$$




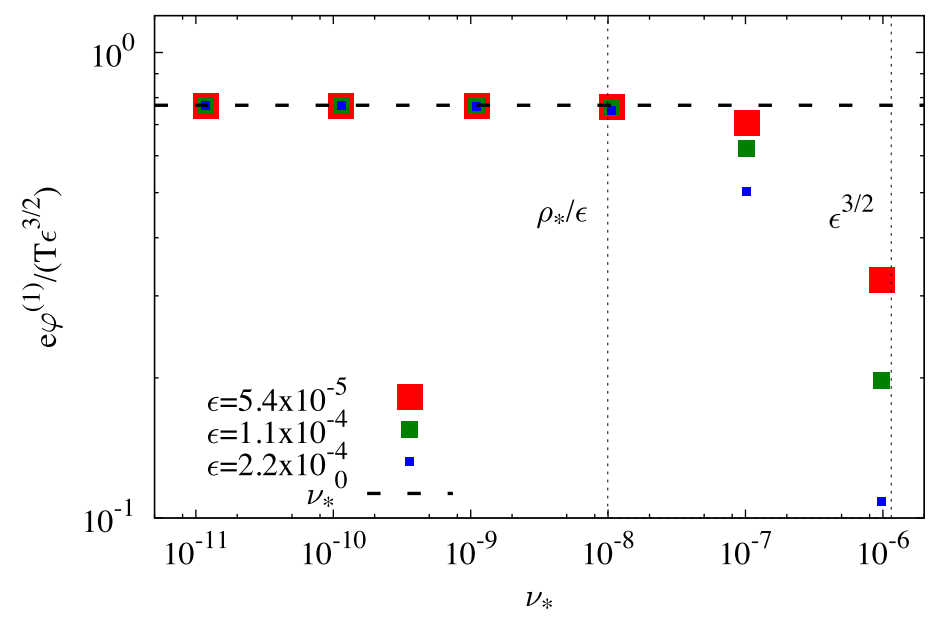

FIGURE 8. Scaling of $\varphi^{(1)}$ with $v_{*}$ and $\epsilon$ (evaluated at the point of the flux surface where it reaches its maximum value) in a range of values of the collisionality where (4.37) is observed. Here, $\varphi_{0}^{\prime}=2 \epsilon^{-1} T /\left(e R_{0}\right)$.

One could formally proceed as done after equation (4.17), but it seems difficult to find a completely explicit solution for $\varphi^{(1)}$ due to the relatively complicated dependence of $\partial_{r} J^{(0)}$ on $v$ and $\lambda$. In any case, it is clear that imposing (4.40) implies

$$
J_{\varphi}^{(1)} \sim \epsilon^{1 / 2} v_{t} R_{0}
$$

and, therefore,

$$
\varphi^{(1)} \sim \epsilon \frac{T}{e} .
$$

In addition, from (4.40) we can infer that $\varphi^{(1)}$ is stellarator symmetric.

Note that for $\nu_{*} \sim \rho_{*}$, expression (4.42) matches (4.24).

\subsection{Superbanana-plateau regime}

In this subsection, we treat cases with $\nu_{*} \ll \rho_{*} \ll 1$ and such that $\partial_{r} J^{(0)}$ vanishes for $v \lesssim v_{t}$. Sections 4.1 and 4.2, although brief, were self-contained to a large extent. In this subsection we will refer the reader to Calvo et al. (2017) more frequently for the derivation of particularly technical results.

The zeroes of $\partial_{r} J^{(0)}$ correspond to 'resonant' points of phase space where the orbitaveraged tangential component of the magnetic and $E \times B$ drifts cancel each other.

Recall equation (2.19). The condition $\partial_{r} J^{(0)}=0$ can be compactly expressed as

$$
\lambda \overline{\partial_{r} B_{0}}(r, \lambda)=-\frac{2 Z e \varphi_{0}^{\prime}(r)}{m v^{2}},
$$

where

$$
\overline{(\cdot)}=\frac{1}{\tau_{b}^{(0)}} \sum_{\sigma} \int_{l_{b_{10}}}^{l_{b_{20}}}(\cdot)\left|v_{\|}^{(0)}\right|^{-1} \mathrm{~d} l
$$

denotes orbit average and

$$
\tau_{b}^{(0)}(r, v, \lambda)=2 \int_{l_{b_{10}}}^{l_{b_{20}}}\left|v_{\|}^{(0)}\right|^{-1} \mathrm{~d} l
$$


is the time that it takes for the particle to complete the orbit in the magnetic field $B_{0}$. A necessary (but not sufficient) condition for this equation to have solutions for $v \lesssim v_{t}$ is

$$
\frac{Z e\left|\varphi_{0}^{\prime}\right|}{T} \lesssim \frac{1}{R_{0}}
$$

That is, the effect of the resonances is expected to be important for relatively small values of the radial electric field. When $v_{*} \ll \rho_{*} \ll 1$ and $Z e\left|\varphi_{0}^{\prime}\right| / T \gg R_{0}^{-1}$, the treatment of subsection (4.2) applies.

From here on, let us assume $v_{*} \ll \rho_{*} \ll 1$ and condition (4.46) in the rest of this subsection, and let us also assume that, for $v \lesssim v_{t}$, there exists one solution in $\lambda$ of the resonance condition (4.43), that we denote by $\lambda_{r}\left(r, v, \varphi_{0}^{\prime}\right)$. In general, $\lambda_{r}$ will be defined only for some values of $r, v$ and $\varphi_{0}^{\prime}$. For fixed $r$ and $\varphi_{0}^{\prime}$, we denote by $I$ the interval in $v$ for which $\lambda_{r}$ is defined.

We can expand the drift-kinetic equation (2.18) around the position of the resonance. Defining $\xi=\lambda-\lambda_{r}\left(r, v, \varphi_{0}^{\prime}\right)$ and writing the drift-kinetic equation in coordinates $v$ and $\xi$, we have

$$
\partial_{\lambda} \partial_{r} J_{r}^{(0)} \xi \partial_{\alpha} g_{\mathrm{rl}}+k \partial_{\xi}^{2} g_{\mathrm{rl}}=\left(\partial_{\alpha} J_{B, r}^{(1)}+\partial_{\alpha} \widehat{J_{\varphi}^{(1)}}\right) \Upsilon F_{M},
$$

where $k(r, v)$ is given by

$$
k=\frac{2 Z e \Psi}{m v}\left[v_{\lambda} \lambda_{r} \int_{l_{b_{10}}}^{l_{b_{20}}} B_{0}^{-1} \sqrt{1-\lambda_{r} B_{0}} \mathrm{~d} l+\frac{\nu_{v} v^{2}}{2}\left(\partial_{v} \lambda_{r}\right)^{2} \int_{l_{b_{10}}}^{l_{b_{20}}} \frac{1}{\sqrt{1-\lambda_{r} B_{0}}} \mathrm{~d} l\right]
$$

subindices $r$ indicate that the corresponding quantity is evaluated at $\lambda=\lambda_{r}\left(r, v, \varphi_{0}^{\prime}\right)$ and $\widehat{J_{\varphi}^{(1)}}$ is an approximation of $J_{\varphi}^{(1)}$ around $\lambda_{r}$. In particular, $\widehat{J_{\varphi}^{(1)}}$ is a function of $v$ and $\xi$. We will see shortly why, in general, we cannot simply evaluate $J_{\varphi}^{(1)}$ at $\lambda_{r}$. The second term in (4.48) comes from the energy diffusion piece of the collision operator, which must be included because the coordinate across the layer (the coordinate in which $g_{\mathrm{rl}}$ varies fast), $\xi=\lambda-\lambda_{r}$, mixes $v$ and $\lambda$. The collision frequency $v_{v}$ is given by

$$
v_{v}(v)=\frac{3 \sqrt{\pi} \chi(v \sqrt{m /(2 T)})}{2 \tau_{i i}(v \sqrt{m /(2 T)})^{3}} .
$$

In Calvo et al. (2017), the energy diffusion piece of the collision operator was incorrectly neglected. Note that none of the scalings derived in Calvo et al. (2017) are affected by the inclusion of energy diffusion.

Using that $\partial_{\lambda} \partial_{r} J_{r}^{(0)}=O\left(B_{0} v_{t}\right)$ and that $k(r, v)=O\left(v_{\lambda} B_{0}^{-2} R_{0} \rho_{*}^{-1}\right)$, and balancing the two terms on the left-hand side of (4.47), we deduce that $g_{\mathrm{rl}}$ is localized in a layer whose size in the coordinate $\xi$ is

$$
B_{0} \Delta_{\mathrm{sb}-\mathrm{p}} \sim\left(v_{*} / \rho_{*}\right)^{1 / 3} .
$$

Then, balancing the left- and right-hand sides of (4.47), we find that

$$
g_{\mathrm{rl}} \sim\left(B_{0} \Delta_{\mathrm{sb}-\mathrm{p}}\right)^{-1} F_{M} .
$$

This resonant layer, via $g_{\mathrm{rl}}$, is responsible for superbanana-plateau transport. 
The solution outside the resonant layer is given by (4.30). Since $g_{0}$ diverges at $\lambda_{r}$, we remove the divergence so that we obtain a function $g_{0}^{\text {out }}$ which is finite everywhere and asymptotically coincides with $g_{0}$ far from the resonant layer. Namely,

$$
g_{0}^{\text {out }}=g_{0}-\frac{1}{\left(\lambda-\lambda_{r}\right) \partial_{\lambda} \partial_{r} J_{r}^{(0)}}\left(\widetilde{J}_{B, r}^{(1)}+\widehat{J_{\varphi}^{(1)}}-\frac{1}{2 \pi} \int_{0}^{2 \pi} \widehat{J_{\varphi}^{(1)}} \mathrm{d} \alpha\right) \Upsilon F_{M},
$$

where $\widetilde{J}_{B, r}^{(1)}$ is the quantity defined in (4.35) evaluated at $\lambda_{r}$.

In principle, both pieces, $g_{0}^{\text {out }}$ and $g_{\mathrm{rl}}$, contribute equally to the quasineutrality equation (although only $g_{\mathrm{rl}}$ contributes to radial transport). Hence, one needs to solve

$$
\varphi^{(1)}=\frac{2 \pi B_{0}}{e n}\left(\frac{Z}{T}+\frac{1}{T_{e}}\right)^{-1}\left[\int_{0}^{\infty} \mathrm{d} v \int_{B_{0, \max }^{-1}}^{B^{-1}} \mathrm{~d} \lambda \frac{v^{3} g_{0}^{\text {out }}}{\left|v_{\|}^{(0)}\right|}+\int_{I} \mathrm{~d} v \int_{K \Delta_{\mathrm{sb}-\mathrm{p}}} \mathrm{d} \lambda \frac{v^{3} g_{\mathrm{rl}}}{\left|v_{\|}^{(0)}\right|}\right],
$$

with $K \gg 1$. An explicit, asymptotically correct expression for the quasineutrality equation is given by (see Calvo et al. (2017) for the details)

$$
\begin{aligned}
\varphi^{(1)}= & \frac{2 \pi B_{0}}{e n}\left(\frac{Z}{T}+\frac{1}{T_{e}}\right)^{-1}\left\{\int_{0}^{\infty} \mathrm{d} v \int_{B_{0, \max }^{-1}}^{B^{-1}} \mathrm{~d} \lambda \frac{v^{3}}{\left|v_{\|}^{(0)}\right|} g_{0}^{\text {out }}\right. \\
& +\int_{I} \mathrm{~d} v v^{2} \int_{-\infty}^{\lambda_{L}(l)} \mathrm{d} \lambda \frac{g_{\mathrm{rl}}}{\sqrt{\lambda_{r}\left|\partial_{l} B_{0}\left(l_{L}\right)\right|\left(l-l_{L}\right)-\left(\lambda-\lambda_{r}\right) B_{0}\left(l_{L}\right)}} \\
& +\int_{I} \mathrm{~d} v v^{2} \int_{-\infty}^{\lambda_{R}(l)} \mathrm{d} \lambda \frac{g_{\mathrm{rl}}}{\sqrt{\lambda_{r}\left|\partial_{l} B_{0}\left(l_{R}\right)\right|\left(l_{R}-l\right)-\left(\lambda-\lambda_{r}\right) B_{0}\left(l_{R}\right)}} \\
& +\int_{I} \mathrm{~d} v v^{2}\left[\frac{1}{\sqrt{1-\lambda_{r} B_{0}(l)}}-\frac{1}{\sqrt{\lambda_{r}\left|\partial_{l} B_{0}\left(l_{L}\right)\right|\left(l-l_{L}\right)}}\right. \\
& \left.\left.-\frac{1}{\sqrt{\lambda_{r}\left|\partial_{l} B_{0}\left(l_{R}\right)\right|\left(l_{R}-l\right)}}\right] \int_{-\infty}^{\infty} \mathrm{d} \lambda g_{\mathrm{rl}}\right\}
\end{aligned}
$$

where $l_{L}$ and $l_{R}$ denote the bounce points of the trajectory $\lambda=\lambda_{r}$,

$$
\lambda_{L}(l)-\lambda_{r}=\frac{\lambda_{r}\left|\partial_{l} B_{0}\left(l_{L}\right)\right|\left(l-l_{L}\right)}{B_{0}\left(l_{L}\right)}
$$

and

$$
\lambda_{R}(l)-\lambda_{r}=\frac{\lambda_{r}\left|\partial_{l} B_{0}\left(l_{R}\right)\right|\left(l_{R}-l\right)}{B_{0}\left(l_{R}\right)} .
$$

Expression (4.54) is useful in order to explain why we $\operatorname{did}$ not evaluate $J_{\varphi}^{(1)}$ at $\lambda_{r}$ in (4.47). First, note that the first term in brackets in the fourth line of (4.54) diverges at $l=l_{L}$ and $l=l_{R}$. We distinguish two cases:

(i) If

$$
\left|\frac{2 Z e \varphi_{0}^{\prime}}{m v^{2} \lambda_{r} \partial_{\lambda} \overline{\partial_{r} B_{0}}\left(\lambda_{r}\right)}\right| \gg \Delta_{\mathrm{sb}-\mathrm{p}},
$$

the dependence of $\lambda_{r}$ on $v$ is strong enough for the integral over $v$ to smooth out the divergence of $1 / \sqrt{1-\lambda_{r} B_{0}(l)}$ at $l=l_{L}$ and $l=l_{R}$. Then,

$$
\varphi^{(1)} \sim \frac{T}{e} .
$$




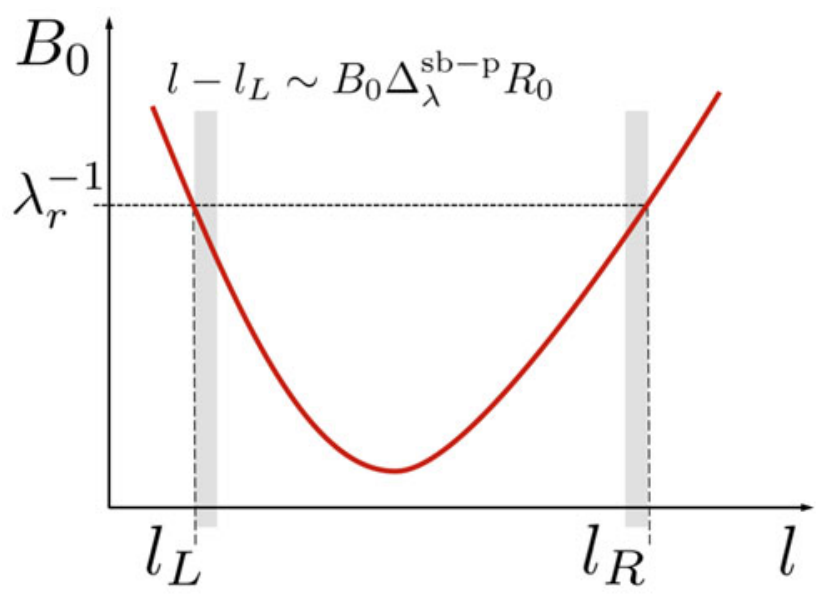

FIGURE 9. If (4.60) is satisfied, the resonant value $\lambda_{r}$ is approximately independent of $v$. The accumulated effect of the resonance for all possible values of $v$ produces a larger $\varphi^{(1)}$ in a neighbourhood of the bounce points of the resonant trajectory, $l_{L}$ and $l_{R}$.

In this case, $\widehat{J_{\varphi}^{(1)}}$ can be replaced by $J_{\varphi, r}^{(1)}$ in $(4.47)$, where

$$
J_{\varphi, r}^{(1)}=-\frac{2 Z e}{m v} \int_{l_{b_{10}}}^{l_{b_{20}}} \frac{\varphi^{(1)}}{\sqrt{1-\lambda_{r} B_{0}}} \mathrm{~d} l,
$$

and the solution of (4.47) can be found analytically, with $g_{\mathrm{rl}}$ approaching a Dirac delta distribution when the collision frequency tends to zero. This gives the standard superbanana-plateau regime in which the radial neoclassical fluxes are strictly independent of the collisionality. This regime was treated in Shaing (2015) for tokamaks of finite aspect ratio with broken symmetry.

(ii) If

$$
\left|\frac{2 Z e \varphi_{0}^{\prime}}{m v^{2} \lambda_{r} \partial_{\lambda} \overline{\partial_{r} B_{0}}\left(\lambda_{r}\right)}\right| \ll \Delta_{\mathrm{sb}-\mathrm{p}},
$$

then $\lambda_{r}$ is approximately independent of $v$ and no such smoothing happens. The effect of the accumulation of the resonances at the same $\lambda_{r}$ for every $v$ means that, close to the bounce points of the resonant trajectory, the electrostatic potential becomes very large. Specifically, one finds that

$$
\varphi^{(1)} \sim \begin{cases}\left(v_{*} / \rho_{*}\right)^{-1 / 6} T / e & \left|l-l_{j}\right| \sim B_{0} \Delta_{\mathrm{sb}-\mathrm{p}} R_{0} \\ T / e & \left|l-l_{j}\right| \gg B_{0} \Delta_{\mathrm{sb}-\mathrm{p}} R_{0},\end{cases}
$$

where $j=L, R$ (see figure 9). This subregime of the superbanana-plateau regime was discovered in Calvo et al. (2017) and we will focus on it in the next subsection, devoted to illustrating it numerically. When (4.60) holds, the radial neoclassical fluxes depend logarithmically on collisionality. The estimation (4.50) for the size of the layer also incorporates logarithmic corrections.

Finally, we point out that, as clearly inferred from inspection of (4.47), $g_{\mathrm{rl}}$ has no definite parity under stellarator symmetry transformations, and neither does $\varphi^{(1)}$. 


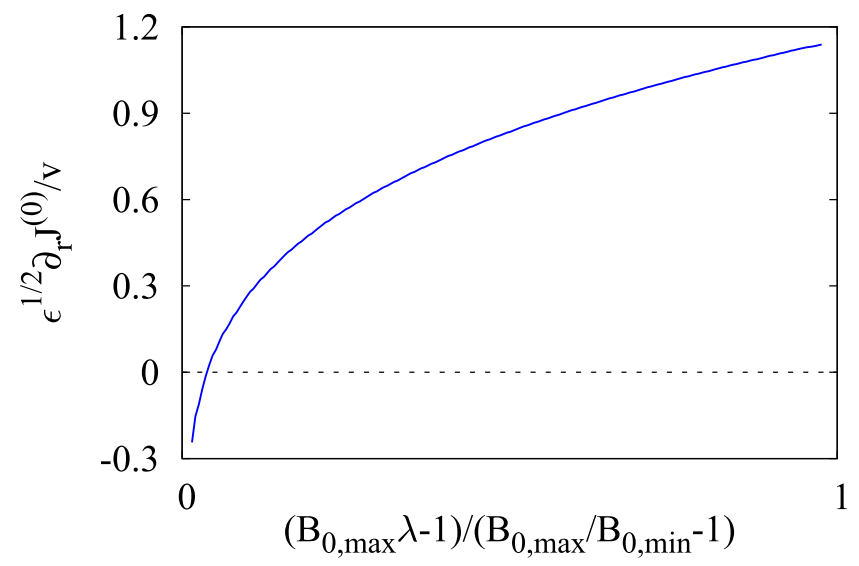

FIgURE 10. Plot of $\partial_{r} J^{(0)}$ as a function of $\lambda$ in our model omnigenous magnetic field, with $\epsilon=1.1 \times 10^{-4}$ and $\varphi_{0}^{\prime}=0$. The value of $\lambda$ at which $\partial_{r} J^{(0)}$ vanishes determines the resonant value $\lambda_{r}$. Here, $\left(B_{0, \max } \lambda_{r}-1\right) /\left(B_{0, \max } / B_{0, \min }-1\right)=0.04$.

(a)

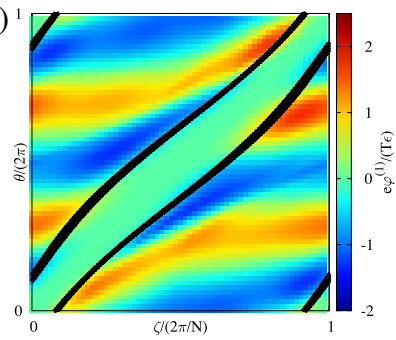

(b)

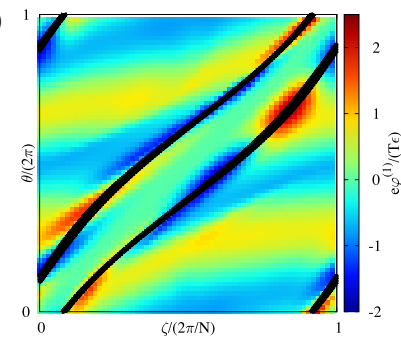

(c)

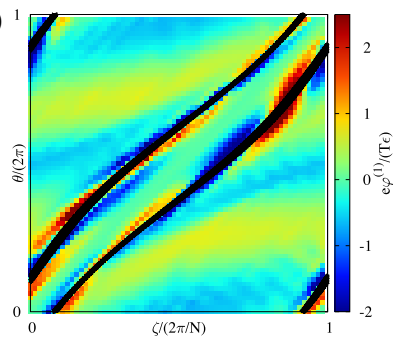

FIgURE 11. Contour plot of $\varphi^{(1)}$ for $\epsilon=1.1 \times 10^{-4}, \varphi_{0}^{\prime}=0$ and three values of the collisionality. From left to right, $v_{*}=1.2 \times 10^{-12}, v_{*}=1.3 \times 10^{-13}, v_{*}=1.3 \times 10^{-14}$. The black lines are the two curves determined by the left and right bounce points of the resonant trajectory, $l_{L}$ and $l_{R}$, respectively. When $v_{*}$ decreases, the increase of $\varphi^{(1)}$ around $l_{L}$ and $l_{R}$, as predicted by (4.67), is confirmed. The colour scale has been kept constant in the three panels, so that the increase in $\varphi^{(1)}$ becomes clearer; that is, in $(b, c), e\left|\varphi^{(1)}\right| /(T \epsilon)$ reaches larger values than the largest one indicated in the colour scale bar.

\subsubsection{Superbanana-plateau regime in a large aspect ratio stellarator}

We start by giving some estimates that are needed below. First, note that $\partial_{\lambda} \partial_{r} J_{r}^{(0)} \sim$ $\epsilon^{-3 / 2} v_{t} B_{00}$. Second, observe that for large aspect ratio $\partial_{v} \lambda_{r} \sim \epsilon /\left(B_{00} v_{t}\right)$ so that the term proportional to $v_{v}$ in (4.48) can be neglected compared to the term proportional to $v_{\lambda}$, giving $k \sim \epsilon^{3 / 2} v_{\lambda} B_{00}^{-2} R_{0} \rho_{*}^{-1}$. Then, balancing the two terms on the left-hand side of the drift-kinetic equation (4.47), we find that, for $\epsilon \ll 1$,

$$
B_{00} \Delta_{\mathrm{sb}-\mathrm{p}} \sim \epsilon\left(\frac{\nu_{*}}{\rho_{*}}\right)^{1 / 3} .
$$

For convenience, define $g_{\mathrm{rl}}\left[J_{B}^{(1)}\right]$ and $g_{\mathrm{rl}}\left[J_{\varphi}^{(1)}\right]$ as the solutions of

$$
\partial_{\lambda} \partial_{r} J_{r}^{(0)} \xi \partial_{\alpha} g_{\mathrm{rl}}\left[J_{B}^{(1)}\right]+k \partial_{\xi}^{2} g_{\mathrm{rl}}\left[J_{B}^{(1)}\right]=\partial_{\alpha} J_{B, r}^{(1)} \Upsilon F_{M}
$$




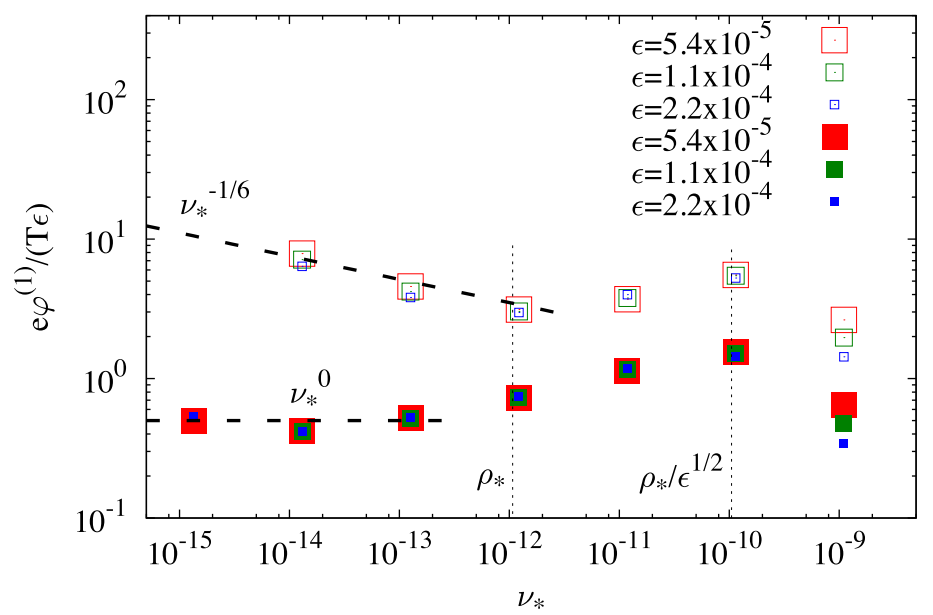

FIgURE 12. Size of $\varphi^{(1)}$ as a function of $\nu_{*}$ and $\epsilon$. Here, $\varphi_{0}^{\prime}=0$. The two scalings given in (4.67) are checked. Full squares give the size of $\varphi^{(1)}$ at generic points, $\left|l-l_{j}\right| \gg B_{00} \Delta_{\mathrm{sb}-\mathrm{p}} R_{0}$, whereas empty squares give the size of $\varphi^{(1)}$ at a point that is very close to a bounce point of the resonant trajectory, $\left|l-l_{j}\right| \sim B_{00} \Delta_{\mathrm{sb}-\mathrm{p}} R_{0}$ (that is, empty squares give approximately the maximum of $\varphi^{(1)}$ on the flux surface).

and

$$
\partial_{\lambda} \partial_{r} J_{r}^{(0)} \xi \partial_{\alpha} g_{\mathrm{rl}}\left[J_{\varphi}^{(1)}\right]+k \partial_{\xi}^{2} g_{\mathrm{rl}}\left[J_{\varphi}^{(1)}\right]=\partial_{\alpha} \widehat{J_{\varphi}^{(1)}} \Upsilon F_{M}
$$

respectively. Of course, $g_{\mathrm{rl}}=g_{\mathrm{rl}}\left[J_{B}^{(1)}\right]+g_{\mathrm{rl}}\left[J_{\varphi}^{(1)}\right]$. Then,

$$
g_{\mathrm{rl}}\left[J_{B}^{(1)}\right] \sim\left(\frac{v_{*}}{\rho_{*}}\right)^{-1 / 3} \frac{n}{v_{t}^{3}}
$$

and

$$
g_{\mathrm{rl}}\left[J_{\varphi}^{(1)}\right] \sim \epsilon^{-1 / 2}\left(\frac{\nu_{*}}{\rho_{*}}\right)^{-1 / 3} \frac{J_{\varphi}^{(1)}}{v_{t} R_{0}} \frac{n}{v_{t}^{3}} .
$$

One can deal with the quasineutrality equation (4.54) by employing again the procedure followed after (4.17) and also in $\S 4.2 .2$. The result is that

$$
\varphi^{(1)} \sim \begin{cases}\left(v_{*} / \rho_{*}\right)^{-1 / 6} \epsilon T / e & \left|l-l_{j}\right| \sim B_{00} \Delta_{\mathrm{sb}-\mathrm{p}} R_{0} \\ \epsilon T / e & \left|l-l_{j}\right| \gg B_{00} \Delta_{\mathrm{sb}-\mathrm{p}} R_{0}\end{cases}
$$

Let us check numerically the scalings of this subsection. First, in figure 10, we show the dependence of $\partial_{r} J^{(0)}$ on $\lambda$ for $\varphi_{0}^{\prime}=0$ in our model magnetic configuration and we give, in particular, the location in phase space of the resonant value $\lambda_{r}$. In figure 11, contour plots of $\varphi^{(1)}$ are shown for three different values of the collisionality, so that one can see how $\varphi^{(1)}$ becomes larger and larger around $l_{L}$ and $l_{R}$ as the collisionality decreases. Figure 12 provides a precise numerical check of the two scalings given in (4.67). 


\section{Conclusions}

The component of the neoclassical electrostatic potential that is non-constant on flux surfaces, $\tilde{\varphi}$ (equivalently, the component of the electric field that is tangential to the surface), has been proven to have an important impact on stellarator radial impurity transport (García-Regaña et al. 2013), triggering interest in the correct calculation of the tangential electric field, which has often been neglected in stellarator neoclassical theory. On the numerical side, remarkable effort has been made during recent years (see García-Regaña et al. (2017) and references therein). In this article, we have studied analytically the scaling of $\tilde{\varphi}$ with collisionality and aspect ratio, and its structure on the flux surface.

The tangential electric field can be remarkably large when the collisionality is low enough for the tangential drifts to count in the drift-kinetic equation and especially when the tangential magnetic drift is non-negligible. If the collisionality and the radial electric field are such that the tangential magnetic drift cannot be dropped, the neoclassical equations can be kept linear and radially local only if the stellarator is sufficiently optimized (Calvo et al. 2017); i.e. if the magnetic configuration is sufficiently close to omnigeneity (Cary \& Shasharina 1997; Parra et al. 2015). This is why we have conducted our study in the framework of a stellarator that is close to being omnigenous, employing the techniques developed in Calvo et al. (2017), where a small parameter $\delta$ exists that gives the size of the deviation from perfect omnigeneity. Throughout the paper, and employing some results of Calvo et al. (2017), we discuss the calculation of the tangential electric field for collisionality values that cover the $1 / \nu, \sqrt{v}$ and superbanana-plateau regimes, and in each of them provide its size, scaling with collisionality, aspect ratio and $\delta$ and behaviour under stellarator symmetry transformations. In the treatment presented in Calvo et al. (2017) no assumption was made about the aspect ratio of the stellarator. Here, we have investigated how the results are modified when, apart from the $\delta$ expansion, one takes a subsidiary expansion in large aspect ratio. We have found new regimes that did not appear in tight aspect ratio devices. In particular, we have deduced that the maximum size admitted for $\tilde{\varphi}:=\delta \varphi^{(1)}$ by the neoclassical equations is given by $\varphi^{(1)} \sim \epsilon T / e$, except at some special points where it can be larger. Table 1 summarizes the analytical results, providing the scaling with collisionality and aspect ratio in each regime. The notation is explained in $\$ \S 2$ and 4 .

The analytical results for each asymptotic regime have been verified and illustrated by numerical calculations of the code KNOSOS, that solves the equations derived in Calvo et al. (2017) for stellarators close to omnigeneity, the same set of equations that are the subject of study of this paper. In figure 3, choosing realistic values for a stellarator reactor plasma, we have represented the size of $\varphi^{(1)}$ as a function of the collisionality, showing the different regimes in a single figure.

Finally, we comment on the expected effect of $\tilde{\varphi}$ on the radial neoclassical transport of the main ions. This effect is negligible compared to the transport due to $B_{1}$ except in regimes where $\tilde{\varphi}$ reaches its maximum size, given by $\varphi^{(1)} \sim \epsilon T / e$ (see table 1 ). In principle, in such regimes, the contributions of $B_{1}$ and $\tilde{\varphi}$ to the main ion neoclassical fluxes can be comparable. In practice, however, the maximum size reached by $\tilde{\varphi}$ is somewhat smaller. Obtaining $\varphi^{(1)} \sim \epsilon T / e$ seems to require density profile gradients $\left|n^{\prime} / n\right| \sim\left(\epsilon R_{0}\right)^{-1}$, like the ones employed in this paper. If one takes a smaller density profile gradient and $\left|T^{\prime} / T\right| \sim\left(\epsilon R_{0}\right)^{-1}$ (a situation that is more common in stellarators), the scaling of $\tilde{\varphi}$ does not change but the actual value is smaller, due to the different way in which the density and temperature profiles enter the source term of the driftkinetic equation. 


\begin{tabular}{|c|c|c|c|}
\hline Parameter range & Size of $e \varphi^{(1)} / T$ & $\begin{array}{l}\text { Behaviour under } \\
\text { stellarator } \\
\text { symmetry }\end{array}$ & $\begin{array}{l}\text { Radial transport } \\
\text { regime }\end{array}$ \\
\hline \multicolumn{4}{|c|}{ Large radial electric field, $\left|\varphi_{0}^{\prime}\right| \sim \epsilon^{-1} T /\left(e R_{0}\right)$} \\
\hline $\begin{array}{l}\epsilon^{-1} \rho_{*} \ll \nu_{*} \ll \epsilon^{3 / 2} \\
\nu_{*} \ll \epsilon^{-1} \rho_{*}\end{array}$ & $\begin{array}{l}\epsilon^{1 / 2} \rho_{*} / \nu_{*} \\
\epsilon^{3 / 2}\end{array}$ & $\begin{array}{l}\text { Antisymmetric } \\
\text { Symmetric }\end{array}$ & $\begin{array}{l}1 / v \\
\sqrt{v}\end{array}$ \\
\hline
\end{tabular}

Small radial electric field, $\left|\varphi_{0}^{\prime}\right| \lesssim T /\left(e R_{0}\right)$

\begin{tabular}{l|l|l|l}
$\epsilon^{-1 / 2} \rho_{*} \ll v_{*} \ll \epsilon^{3 / 2}$ & $\epsilon^{1 / 2} \rho_{*} / \nu_{*}$ & Antisymmetric & $1 / v$ \\
$\rho_{*} \ll v_{*} \ll \epsilon^{-1 / 2} \rho_{*}$ & $\epsilon$ & Symmetric & $1 / v$ \\
$\nu_{*} \ll \rho_{*}$ & $\epsilon$ & Symmetric & $\sqrt{\nu}$ \\
$\left(\partial_{r} J^{(0)}\right.$ has no zeros $)$ & & No defined symmetry & Superbanana plateau \\
$\nu_{*} \ll \rho_{*}$ & $\epsilon$, at generic points \\
$\left(\partial_{r} J^{(0)}\right.$ has zeros $)$ & $\begin{array}{l}\epsilon\left(\nu_{*} / \rho_{*}\right)^{-1 / 6}, \text { at } \\
\text { special points, if any }\end{array}$ &
\end{tabular}

TABLE 1. Asymptotic regimes for $\varphi^{(1)}$.

\section{Acknowledgements}

The authors would like to thank an anonymous referee for pointing out that, in general, the energy diffusion piece of the collision operator cannot be neglected in (4.47). This work has been carried out within the framework of the EUROfusion Consortium and has received funding from the Euratom research and training programme 2014-2018 under grant agreement no. 633053. The views and opinions expressed herein do not necessarily reflect those of the European Commission. This research was supported in part by grant ENE2015-70142-P, Ministerio de Economía y Competitividad, Spain.

\section{Appendix A. Application of the Abel transform to integrals over trapped trajectories}

We give a useful inversion formula that interchanges the coordinates $l$ and $\lambda$. In this appendix, the coordinates $r, \alpha$ and $v$ are irrelevant, and we omit them.

For any function $f(l)$, we define $F(\lambda)$ as the following integral over the trapped trajectory determined by $\lambda \in\left[B_{0, \max }^{-1}, B_{0, \min }^{-1}\right]$,

$$
F(\lambda):=\int_{l_{b_{10}}(\lambda)}^{l_{b_{20}}(\lambda)} \frac{f(l)}{\sqrt{1-\lambda B_{0}(l)}} \mathrm{d} l .
$$

Then, given a value of the magnetic field $\hat{B} \in\left[B_{0, \min }, B_{0, \max }\right]$, we have

$$
\left.\frac{f}{\left|\partial_{l} B_{0}\right|}\right|_{l_{b_{20}}\left(\hat{B}^{-1}\right)}+\left.\frac{f}{\left|\partial_{l} B_{0}\right|}\right|_{l_{b_{10}}\left(\hat{B}^{-1}\right)}=-\frac{1}{\pi \hat{B}} \int_{\hat{B}^{-1}}^{B_{0, \min }^{-1}} \frac{\mathrm{d} F}{\mathrm{~d} \lambda} \frac{1}{\sqrt{\lambda \hat{B}-1}} \mathrm{~d} \lambda
$$


which can be proven by direct check. This formula can be viewed as a particularization of the Abel transform (Abel 1826).

For the sake of the application of the inversion formula in the main text of this article, we point out the following property, which is immediately derived from (A 2): if $f(l)$ is such that $B_{0}\left(l_{1}\right)=B_{0}\left(l_{2}\right) \Rightarrow f\left(l_{1}\right)=f\left(l_{2}\right)$, then

$$
F(\lambda)=0 \text {, for every } \lambda \Rightarrow f(l)=0 \text {, for every } l .
$$

\section{REFERENCES}

Abel, N. H. 1826 Auflösen einer mechanischen Aufgabe. J. Reine Angew. Math. 1, 153.

Alonso, J. A., Velasco, J. L., Calvo, I., Estrada, T., Fontdecaba, J. M., García-Regaña, J. M., Geiger, J., Landreman, M., McCarthy, K. J., Medina, F. et al. 2016 Parallel impurity dynamics in the TJ-II stellarator. Plasma Phys. Control. Fusion 58, 074009.

Arévalo, J., Alonso, J. A., McCarthy, K. J., Velasco, J. L., García-Regaña, J. M. \& LANDreman, M. 2014 Compressible impurity flow in the TJ-II stellarator. Nucl. Fusion 54, 013008.

Beidler, C. D., Allmaier, K., Isaev, M. Yu., Kasilov, S. V., Kernbichler, W., Leitold, G. O., MaAssberg, H., Miknelsen, D. R., Murakami, S., Schmidt, M. et al. 2011 Benchmarking of the mono-energetic transport coefficients - results from the international collaboration on neoclassical transport in stellarators (ICNTS). Nucl. Fusion 51, 076001.

Boozer, A. H. 1981 Plasma equilibrium with rational magnetic surfaces. Phys. Fluids 24, 1999.

Burhenn, R., Feng, Y., Ida, K., MaAssberg, H., McCarthy, K. J., Kalinina, D., Kobayashi, M., Morita, S., Nakamura, Y., Nozato, H. et al. 2009 On impurity handling in high performance stellarator/heliotron plasmas. Nucl. Fusion 49, 065005.

Calvo, I., Parra, F. I., Velasco, J. L. \& Alonso, J. A. 2013 Stellarators close to quasisymmetry. Plasma Phys. Control. Fusion 55, 125014.

Calvo, I., Parra, F. I., Alonso, J. A. \& Velasco, J. L. 2014 Optimizing stellarators for large flows. Plasma Phys. Control. Fusion 56, 094003.

Calvo, I., Parra, F. I., Velasco, J. L. \& Alonso, J. A. 2015 Flow damping in stellarators close to quasisymmetry. Plasma Phys. Control. Fusion 57, 014014.

Calvo, I., Parra, F. I., Velasco, J. L. \& Alonso, J. A. 2017 The effect of tangential drifts on neoclassical transport in stellarators close to omnigeneity. Plasma Phys. Control. Fusion 59, 055014.

Calvo, I., Parra, F. I., Velasco, J. L., Alonso, J. A. \& García-Regaña, J. M. 2018 Stellarator impurity flux driven by electric fields tangent to magnetic surfaces. arXiv:1803.05691.

CARY, J. R. \& Shasharina, S. G. 1997 Omnigenity and quasihelicity in helical plasma confinement systems. Phys. Plasmas 4, 3323.

Dewar, R. L. \& Hudson, S. R. 1998 Stellarator symmetry. Physica D 112, 275.

Galeev, A. A. \& SAgdeev, R. Z. 1979 Theory of neoclassical diffusion. In Reviews of Plasma Physics (ed. M. A. Leontovich), vol. 7, p. 257. Consultants Bureau.

García-Regaña, J. M., Kleiber, R., Beidler, C. D., Turkin, Y., MaAssberg, H. \& Helander, P. 2013 On neoclassical impurity transport in stellarator geometry. Plasma Phys. Control. Fusion 55, 074008.

García-Regaña, J. M., Beidler, C. D., Kleiber, R., Helander, P., Mollén, A., Alonso, J. A., Landreman, M., MaAssberg, H., Smith, H. M., Turkin, Y. et al. 2017 Electrostatic potential variation on the flux surface and its impact on impurity transport. Nucl. Fusion 57, 056004.

García-Regaña, J. M., Estrada, T., Calvo, I., Velasco, J. L., Alonso, J. A., Carralero, D., Kleiber, R., Landreman, M., Mollén, A., Sánchez, E. et al. 2018 On-surface potential and radial electric field variations in electron root stellarator plasmas. arXiv:1804.10424. 
Hall, L. S. \& MCNAmara, B. 1975 Three-dimensional equilibrium of the anisotropic, finite-pressure guiding-center plasma: theory of the magnetic plasma. Phys. Fluids 18, 552.

Hazeltine, R. D. 1973 Recursive derivation of drift-kinetic equation. Plasma Phys. 15, 77.

Helander, P. \& Sigmar, D. J. 2002 Collisional Transport in Magnetized Plasmas (ed. M. G. Haines et al.), Cambridge Monographs on Plasma Physics. Cambridge University Press.

Helander, P. \& NÜHREnberg, J. 2009 Bootstrap current and neoclassical transport in quasiisodynamic stellarators. Plasma Phys. Control. Fusion 51, 055004.

Helander, P., Newton, S. L., Mollén, A. \& Smith, H. M. 2017 Impurity transport in a mixed-collisionality stellarator plasma. Phys. Rev. Lett. 118, 155002.

Ho, D. D. M. \& Kulsrud, R. M. 1987 Neoclassical transport in stellarators. Phys. Fluids 30, 442.

Ida, K., Yoshinuma, M., Osakabe, M., Nagaoka, K., Yokoyama, M., Funaba, H., Suzuki, C., Ido, T., Shimizu, A., Murakami, I. et al. 2009 Observation of an impurity hole in a plasma with an ion internal transport barrier in the large helical device. Phys. Plasmas 16, 056111.

Klinger, T., Alonso, A., Bozhenkov, S., Burhenn, R., Dinklage, A., Fuchert, G., Geiger, J., Grulke, O., Langenberg, A., Hirsch, M. et al. 2017 Performance and properties of the first plasmas of Wendelstein 7-X. Plasma Phys. Control. Fusion 59, 014018.

Kornilov, V., Kleiber, R. \& HATZKy, R. 2005 Gyrokinetic global electrostatic ion-temperaturegradient modes in finite $\beta$ equilibria of Wendelstein 7-X. Nucl. Fusion 45, 238.

Landreman, M. \& CATto, P. J. 2012 Omnigenity as generalized quasisymmetry. Phys. Plasmas 19, 056103.

Landreman, M., Smith, H. M., Mollén, A. \& Helander, P. 2014 Comparison of particle trajectories and collision operators for collisional transport in nonaxisymmetric plasmas. Phys. Plasmas 21, 042503.

Matsuoka, S., Satake, S., Kanno, R. \& Sugama, H. 2015 Effects of magnetic drift tangential to magnetic surfaces on neoclassical transport in non-axisymmetric plasmas. Phys. Plasmas 22, 072511.

McCormick, K., Grigull, P., Burhenn, R., Brakel, R., Ehmler, H., Feng, Y., Gadelmeier, F., Giannone, L., Hildebrandt, D., Hirsch, M. et al. 2002 New advanced operational regime on the W7-AS stellarator. Phys. Rev. Lett. 89, 015001.

Mollén, A., Landreman, M., Smith, H. M., García-Regaña, J. M. \& Nunami, M. 2018 Flux-surface variations of the electrostatic potential in stellarators: impact on the radial electric field and neoclassical impurity transport. Plasma Phys. Control. Fusion 60, 084001.

MYNICK, H. E. 1984 Calculation of the poloidal ambipolar field in a stellarator and its effect on transport. Phys. Fluids 27, 2086.

PARRA, F. I. \& CALVO, I. 2011 Phase-space Lagrangian derivation of electrostatic gyrokinetics in general geometry. Plasma Phys. Control. Fusion 53, 045001.

Parra, F. I., Calvo, I., Helander, P. \& Landreman, M. 2015 Less constrained omnigeneous stellarators. Nucl. Fusion 55, 033005.

Paul, E. J., Landreman, M., Poli, F. M., Spong, D. A., Smith, H. M. \& Dorland, W. 2017 Rotation and neoclassical ripple transport in ITER. Nucl. Fusion 57, 116044.

Pedrosa, M. A., Alonso, J. A., García-Regaña, J. M., Hidalgo, C., Velasco, J. L., Calvo, I., Kleiber, R., Silva, C. \& Helander, P. 2015 Electrostatic potential variations along flux surfaces in stellarators. Nucl. Fusion 55, 052001.

SHAING, K. C. 2015 Superbanana and superbanana plateau transport in finite aspect ratio tokamaks with broken symmetry. J. Plasma Phys. 81, 905810203.

Velasco, J. L., Calvo, I., Satake, S., Alonso, J. A., Nunami, M., Yokoyama, M., Sato, M., EstradA, T., FontdecabA, J. M., Liniers, M. et al. 2017 Moderation of neoclassical impurity accumulation in high temperature plasmas of helical devices. Nucl. Fusion 57, 016016.

Velasco, J. L., Calvo, i., García-Regaña, J. M., Parra, F. I., Satake, S., Alonso, J. A. \& THE LHD TEAM 2018 Large tangential electric fields in plasmas close to temperature screening. Plasma Phys. Control. Fusion 60, 074004. 